\title{
Invariant subspaces of nilpotent operators and LR-sequences
}

Wing Suet Li and Vladimír Müller

\section{INTRODUCTION}

It is well-known that an operator in a finite-dimensional vector space is uniquely determined up to a similarity by its Jordan model.

Given an operator $T$, two invariant subspaces $\mathcal{M}$ and $\mathcal{N}$ of $T$ are said to be similar if there exists an invertible operator $X$ such that $X T=T X$ and $X \mathcal{M}=\mathcal{N}$. Clearly this is an equivalence relation in the lattice of invariant subspaces of $T$.

Our paper was originally motivated by the following question raised by Bercovici [2].

Problem 0. Given $T \in \mathcal{L}(\mathcal{H})$ and $\mathcal{M} \in \operatorname{Lat}(T)$, describe the similarity orbit of $\mathcal{M}$.

Problem 0 is more complicated than it looks like even in the case of operators on a finite-dimensional space. Using the spectral decomposition it is easy to reduce the problem to the case of nilpotent operators. However, it turns out that there is no Jordan type model for invariant subspaces of finite-dimensional nilpotent operators.

The Jordan model of a nilpotent operator $T$ in a finite-dimensional vector space $\mathcal{H}$ is determined by a partition - a nonincreasing sequence of nonnegative integers designating the sizes of the Jordan blocks of $T$. If $\mathcal{M} \subset \mathcal{H}$ is an invariant subspace for $T$, then, of course, the restriction $T \mid \mathcal{M}$ and the associated quotient operator $\widehat{T}_{\mathcal{M}}$ defined on the quotient space $\mathcal{H} / \mathcal{M}$ are also nilpotent operators. Thus, likewise, the similarity classes of $T \mid \mathcal{M}$ and $\widehat{T}_{\mathcal{M}}$ are determined by such partitions.

In general, partitions corresponding to $T \mid \mathcal{M}$ and $\widehat{T}_{\mathcal{M}}$ do not determine the similarity orbit of $\mathcal{M}$; this is true only in some special cases.

A natural question to ask is what are the possible triples of partitions corresponding to $T \mid \mathcal{M}, \widehat{T}_{\mathcal{M}}$ and $T$. This question, which is surprisingly difficult, has been

1991 Mathematics Subject Classification: Primary 15A21, secondary 15A23, 47A15

Keywords and phrases: LR-sequences, similarity, Jordan model, invariant subspaces of nilpotent operators 
studied over the years from different perspectives. In the group theoretical context, they were studied and solved by Green [6] and Klein [7] in terms of Littlewood-Richardson sequences (LR-sequences). Later, Azenhas and Marques de Sa [1], Thijsse [12] provided constructive solutions of the problems from the matrix polynomial point of view. All of these constructions are rather complicated. For a comprehensive treatment of Littlewood-Richardson sequences, we refer the readers to [8].

The aim of the present paper is to study systematically invariant subspaces of finite-dimensional nilpotent operators. The paper is organized as follows. In Section 1 we recall some of the needed facts from linear algebra and set up the necessary notation. In Section 2 we study the case of cyclic $\widehat{T}_{\mathcal{M}}$ (the Jordan model of $\widehat{T}_{\mathcal{M}}$ consists of a single block). Among others we show that in this case the Jordan model of $T \mid \mathcal{M}$ determines the similarity orbit of $\mathcal{M}$. In Section 3 we study relations between invariant subspaces and various types of LR-sequences. We provide another constructive solution of Klein's result, and at the same time, a better picture of the relation between Littlewood-Richardson sequences and the structure of the associated operator $T$.

In Section 4 we obtain a convexity result about Littlewood-Richardson sequences. The paper concludes in Section 5 with a study of different equivalence classes of invariant suspaces, which envile some interesting properties of the lattice of invariant subspaces of a nilpotent operator on a finite-dimensional vector space.

\section{PRELIMINARIES}

As usual, we denote by $\mathbf{Z}, \mathbf{N}, \mathbf{R}$, and $\mathbf{C}$ the sets of all integers, positive intergers, real, and complex numbers, respectively.

A partition is any (finite or infinite) sequence $\lambda=\left(\lambda_{1}, \lambda_{2}, \ldots\right)$ of nonnegative integers containing only finitely many non-zero terms such that $\lambda_{1} \geq \lambda_{2} \geq \ldots$. We shall not distinguish between two partitions which differ only by a string of zeros at the end. Thus, for example, $(2,1),(2,1,0)$ and $(2,1,0, \ldots)$ are regarded as the same partition. The number of non-zero terms of a partition $\lambda$ is called the length of $\lambda$. Denote further $|\lambda|=\sum_{i} \lambda_{i}$.

The conjugate of a partition $\lambda$ is the partition $\bar{\lambda}=\left(\bar{\lambda}_{1}, \bar{\lambda}_{2}, \ldots\right)$, where $\bar{\lambda}_{j}=\operatorname{card}\left\{i: \lambda_{i} \geq j\right\}$. 
A partition $\lambda$ can be represented by a diagram: a diagram of $\lambda$ is the set of all points $(i, j) \in \mathbf{N}^{2}$ such that $1 \leq j \leq \lambda_{i}$. We are going to use the same symbol $\lambda$ for both a partition and its diagram. Using $\lambda_{i}$ as the length of the $i$-th column (or $\bar{\lambda}_{j}$ as the length of the $j$-th horizontal row), a partition can be conveniently drown into a diagram as follows:

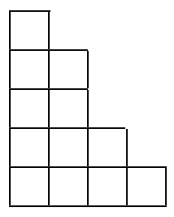

$$
\begin{aligned}
& \lambda=(5,4,2,1) \\
& \bar{\lambda}=(4,3,2,2,1)
\end{aligned}
$$

Figure 1.1

If $\lambda$ and $\mu$ are partitions, we shall write $\mu \subset \lambda$ if the diagram of $\mu$ is contained in the diagram of $\lambda$, i.e., $\mu_{i} \leq \lambda_{i}$ (and $\bar{\mu}_{i} \leq \bar{\lambda}_{i}$ ) for all $i \geq 1$.

By a vector space we mean a finite-dimensional complex vector space; however, all results with the exception of some examples in the last section will remain valid for finite-dimensional vector spaces over any field.

Denote by $\mathcal{L}(\mathcal{H})$ the set of all linear operators in a vector space $\mathcal{H}$. We are going to study only nilpotent operators so that in the following by an operator we always mean a nilpotent operator in a finite-dimensional vector space.

The order of a nilpotent operator $T$ is the smallest interger $n$ such that $T^{n}=0$ and the multiplicity of $T$ is defined to be the smallest cardinality of a set $F$ such that $\mathcal{H}=\vee\left\{T^{n} F: n \geq 0\right\}$. An operator of multiplicity one is called cyclic. Denote by $\mathcal{N}(T)=\{x \in \mathcal{H}: T x=0\}$ the kernel of $T$ and by Lat $(T)$ the lattice of all invariant subspaces of $T$. If $\mathcal{M} \in \operatorname{Lat}(T)$, we denote by $T \mid \mathcal{M}$ the restriction of $T$ to $\mathcal{M}$ and by $\widehat{T}_{\mathcal{M}}: \mathcal{H} / \mathcal{M} \rightarrow \mathcal{H} / \mathcal{M}$ the quotient operator induced by $T$, i.e., $\widehat{T}_{\mathcal{M}}(x+\mathcal{M})=T x+\mathcal{M}$. We say that two operators $T \in \mathcal{L}(\mathcal{H})$ and $S \in \mathcal{L}\left(\mathcal{H}^{\prime}\right)$ are similar (notation: $T \sim S$ ) if there exists an invertible operator $V: \mathcal{H} \rightarrow \mathcal{H}^{\prime}$ such that $V T=S V$.

Let $\lambda$ be a partition and $\mathcal{H}_{\lambda}$ be the vector space with a basis $\left\{e_{i j}: 1 \leq\right.$ $\left.j \leq \lambda_{i}\right\}$. Define operator $S_{\lambda}: \mathcal{H}_{\lambda} \rightarrow \mathcal{H}_{\lambda}$ by $S_{\lambda} e_{i j}=e_{i(j-1)}$ if $j \geq 2$ and $S_{\lambda} e_{i 1}=0$. The operator $S_{\lambda}$ will be called the standard operator of type $\lambda$.

It is well-known that every nilpotent operator $T$ on a finite-dimensional vector space is similar to a standard operator $S_{\lambda}$ (so called the Jordan model of $T$ ). 
We say that $T$ is of type $\lambda$ if it is similar to $S_{\lambda}$. The following is a simple but useful fact.

Lemma 1.1. Let $T \in \mathcal{L}(\mathcal{H})$ be of type $\lambda$. Then

$$
\bar{\lambda}_{i}=\operatorname{dim}\left(\mathcal{N}(T) \cap T^{i-1} \mathcal{H}\right)=\operatorname{dim} \mathcal{N}\left(T^{i}\right)-\operatorname{dim} \mathcal{N}\left(T^{i-1}\right)=\operatorname{dim} T^{i-1} \mathcal{H}-\operatorname{dim} T^{i} \mathcal{H}
$$

The construction of the Jordan model of an operator $T \in \mathcal{L}(\mathcal{H})$ is wellknown. However, we will indicate it here for later use.

Let $T$ be a nilpotent operator on $\mathcal{H}$ and $\mathcal{M} \in \operatorname{Lat}(T)$. We define the "height" of $x \in \mathcal{H}$ with respect to $\mathcal{M}$ to be

$$
h(x, \mathcal{M})=\min \left\{k: T^{k} x \in \mathcal{M}\right\} .
$$

Suppose $T$ is a nilpotent operator on $\mathcal{H}$. Obviously the number $\lambda_{1}=$ $\max \{h(x,\{0\}): x \in \mathcal{H}\}$ can be attained for some $v_{1} \in \mathcal{H}$. Set $\mathcal{M}_{1}=\vee\left\{T^{j} v_{1}: j \geq 0\right\}$. We construct a sequence of vectors $\left\{v_{1}, v_{2}, \ldots\right\} \subset \mathcal{H}$ and subspaces $\left\{\mathcal{M}_{1}, \mathcal{M}_{2}, \ldots\right\} \subset$ Lat $(T)$ inductively: if $\lambda_{i}=\max \left\{h\left(x, \mathcal{M}_{i-1}\right): x \in \mathcal{H}\right\}$ and $v_{i} \in \mathcal{H}$ satisfies $\lambda_{i}=$ $h\left(v_{i}, \mathcal{M}_{i-1}\right)$ then set $\mathcal{M}_{i}=\mathcal{M}_{i-1} \vee\left\{T^{j} v_{i}: j \geq 0\right\}$. Now $\left(\lambda_{1}, \lambda_{2}, \ldots\right)$ is the type of $T$.

The vectors $v_{i} \in \mathcal{H}$ satisfying $h\left(v_{i}, \mathcal{M}_{i-1}\right)=\lambda_{i}$ can be replaced by vectors $w_{i}$ so that $T^{\lambda_{i}} w_{i}=0$. We omit the details here.

Let $\mathcal{M} \in \operatorname{Lat}(T), \mathcal{M} \neq \mathcal{H}$ and set $m=\max \{h(x, \mathcal{M}): x \in \mathcal{H}\}$. Then $T^{-(m-1)} \mathcal{M}=\{x \in \mathcal{H}: h(x, \mathcal{M})<m\}$ is a proper subspace of $\mathcal{H}$. Thus $\{x \in \mathcal{H}:$ $h(x, \mathcal{M})<m\}$ is a subset of $\mathcal{H}$ of the first category (in the unique topology making of $\mathcal{H}$ a topological vector space.)

Corollary 1.2. Let $T \in \mathcal{L}(\mathcal{H})$ be a nilpotent operator and $\left\{\mathcal{M}_{1}, \ldots, \mathcal{M}_{k}\right\} \subset \operatorname{Lat}(T)$. Then there exists $x \in \mathcal{H}$ such that $h\left(x, \mathcal{M}_{i}\right)=\max \left\{h\left(y, \mathcal{M}_{i}\right): y \in \mathcal{H}\right\}$ for each $i=1,2, \ldots, k$. 


\section{ADDING ONE BLOCK}

The notion of similarity between two invariant subspaces of $T$ was introduced in [3]. We recall the definition below.

Definition 2.1. Let $T \in \mathcal{L}(\mathcal{H}), T^{\prime} \in \mathcal{L}\left(\mathcal{H}^{\prime}\right), \mathcal{M} \in \operatorname{Lat}(T)$, and $\mathcal{M}^{\prime} \in \operatorname{Lat}\left(T^{\prime}\right)$. Then $(T, \mathcal{M})$ is similar to $\left(T^{\prime}, \mathcal{M}^{\prime},\right)$ (denoted by $\left.(T, \mathcal{M}) \sim\left(T^{\prime}, \mathcal{M}^{\prime}\right)\right)$ if there exists an invertible operator $V: \mathcal{H} \rightarrow \mathcal{H}^{\prime}$ such that $V T=T^{\prime} V$ and $V \mathcal{M}=\mathcal{M}^{\prime}$.

Clearly if $(T, \mathcal{M}) \sim\left(T^{\prime}, \mathcal{M}^{\prime}\right)$, then the operators $T$ and $T^{\prime}$ are similar. Analogously, the restrictions $T \mid \mathcal{M}$ and $T^{\prime} \mid \mathcal{M}^{\prime}$ are similar, and so are the quotient operators $\widehat{T}_{\mathcal{M}}$ and $\widehat{T}_{\mathcal{M}^{\prime}}^{\prime}$. In general, $T \sim T^{\prime}, T \mid \mathcal{M} \sim T^{\prime} \mathcal{M}^{\prime}$ and $\widehat{T}_{\mathcal{M}} \sim \widehat{T}_{\mathcal{M}^{\prime}}^{\prime}$ will not guarantee $(T, \mathcal{M}) \sim\left(T^{\prime}, \mathcal{M}^{\prime}\right)$. We will explore such examples in Section 5 . However, in some cases, this implication is true. For example, if $T$ and $T^{\prime}$ are uniform (that is all the blocks in the Jordan model of $T$ have the same dimension; in other words, $T$ is of type $(k, k, \ldots, k))$, see [2]. We shall prove that this is also the case when either $T \mid \mathcal{M}$ or $\widehat{T}_{\mathcal{M}}$ is cyclic. These cases are dual to each other, we prove it for the restriction case.

Lemma 2.2. Let $T \in \mathcal{L}(\mathcal{H}), \mathcal{M} \in \operatorname{Lat}(T)$ and let $\left\{m_{1}, m_{2}, \ldots, m_{r}\right\} \in \mathcal{M}$ be a basis of $\mathcal{M}$ satisfying $T m_{t}=m_{t-1}$ for $2 \leq t \leq r$ and $T m_{1}=m_{0}=0$. Suppose $T$ is of type $\nu$ and $\widehat{T}_{\mathcal{M}}$ is of type $\mu$. Then there exist vectors $v_{1}, v_{2}, \ldots, v_{k}$ in $\mathcal{H}$, where $k$ is the length of $\mu$ such that

$$
\left\{T^{j} v_{i}: 1 \leq i \leq k, 0 \leq j \leq \mu_{i}-1\right\} \cup\left\{m_{t}: 1 \leq t \leq r\right\}
$$

is a basis of $\mathcal{H}$ and $T^{\mu_{i}} v_{i}=m_{s_{i}}$ where $s_{i}=\sum_{t=1}^{i}\left(\nu_{t}-\mu_{t}\right)$ for each $i=1, \ldots, k$.

Proof. As indicated in the Preliminaries, we can find $w \in \mathcal{H}$ such that $h(w,\{0\})=\max \{h(x,\{0\}): x \in \mathcal{H}\}=\nu_{1}$ and $h(w, \mathcal{M})=\max \{h(x, \mathcal{M}): x \in \mathcal{H}\}=\mu_{1}$. Set $s_{1}=\nu_{1}-\mu_{1}$. Since $T^{\mu_{1}} w \in \mathcal{M}, T^{s_{1}}\left(T^{\mu_{1}} w\right)=T^{\nu_{1}} w=0$ and $T^{s_{1}+\mu_{1}-1} w \neq 0$, we have $T^{\mu_{1}} w=\sum_{j=1}^{s_{1}} \alpha_{j} m_{j}$, for some $\alpha_{j} \in \mathbf{C}$, with $\alpha_{s_{1}} \neq 0$. Obviously

$$
T^{\mu_{1}}\left(\vee\left\{T w, T^{2} w, \ldots, T^{s_{1}-1} w\right\}\right)=\vee\left\{m_{1}, m_{2}, \ldots, m_{s_{1}-1}\right\}
$$

Therefore it is possible to find $z \in \vee\left\{T w, T^{2} w, \ldots, T^{s_{1}-1} w\right\}$ and $v_{1}=\left(1 / \alpha_{s_{1}}\right) w-z$ such that

$$
h\left(v_{1},\{0\}\right)=h(w,\{0\})=\nu_{1}, \quad h\left(v_{1}, \mathcal{M}\right)=h(w, \mathcal{M})=\mu_{1}, \quad T^{\mu_{1}} v_{1}=m_{s_{1}}
$$


and $\left\{T^{j} v_{1}: 0 \leq j \leq \mu_{1}-1\right\} \cup\left\{m_{t}: 1 \leq t \leq r\right\}$ is a linearly independent set.

We construct vectors $v_{i}$ inductively. Let $i \leq k$ and suppose we have already constructed vectors $v_{t} \in \mathcal{H} \quad(t=1, \ldots, i-1)$ with $T^{\mu_{t}} v_{t}=m_{s_{t}}, h\left(v_{t}, \mathcal{M}_{t-1}\right)=$ $\nu_{t}, h\left(v_{t}, \mathcal{M}_{t-1}+\mathcal{M}\right)=\mu_{t}$, where $\mathcal{M}_{t}=\left\{T^{j} v_{s}: j \geq 0, s=1, \ldots, t\right\}, \mathcal{M} \cap \mathcal{M}_{t}=$ $\vee\left\{m_{1}, \ldots, m_{s_{t}}\right\}$, and the vectors

$$
\left\{T^{j} v_{t}: 1 \leq t \leq i-1,0 \leq j \leq \mu_{t}-1\right\} \cup\left\{m_{t}: 1 \leq t \leq r\right\}
$$

form a basis of $\mathcal{M}_{i-1}+\mathcal{M}$. As $\mathcal{M}_{i-1}+\mathcal{M} \neq \mathcal{H}$, we can find $w \in \mathcal{H}$ such that $h\left(w, \mathcal{M}_{i-1}\right)=\max \left\{h\left(x, \mathcal{M}_{i-1}\right): x \in \mathcal{H}\right\}=\nu_{i}$ and $h\left(w, \mathcal{M}+\mathcal{M}_{i-1}\right)=\max \{h(x, \mathcal{M}+$ $\left.\left.\mathcal{M}_{i-1}\right): x \in \mathcal{H}\right\}=\mu_{i}$. Since $T^{\mu_{i}} w \in \mathcal{M}+\mathcal{M}_{i-1}$, we can write

$$
T^{\mu_{i}} w=\sum_{j=1}^{r} \alpha_{j} m_{j}+\sum_{t=1}^{i-1}\left(\sum_{j=0}^{\mu_{t}-1} \beta_{t j} T^{j} v_{t}\right)
$$

for some $\alpha_{j}, \beta_{t j} \in \mathbf{C}$. Further, $T^{\mu_{l}} w \in \mathcal{M}+\mathcal{M}_{l-1}$, for $l=1, \ldots, i-1$, so that

$$
\begin{aligned}
T^{\mu_{l}} w & =T^{\mu_{l}-\mu_{i}}\left(T^{\mu_{i}} w\right) \\
& =\sum_{j=1}^{r} \alpha_{j} T^{\mu_{l}-\mu_{j}} m_{j}+\sum_{t=1}^{i-1}\left(\sum_{j=0}^{\mu_{t}-1} \beta_{t j} T^{j+\left(\mu_{l}-\mu_{i}\right)} v_{t}\right) \\
& \in \mathcal{M}+\mathcal{M}_{l-1} .
\end{aligned}
$$

It follows from the properties of the vectors $v_{t}$ that if $\beta_{l j} \neq 0$, then $j+\left(\mu_{l}-\mu_{i}\right) \geq \mu_{l}$, or $\mu_{i} \leq j$. Thus, we can express $T^{\mu_{i}} w$ as

$$
T^{\mu_{i}} w=\sum_{j=1}^{r} \alpha_{j} m_{j}+T^{\mu_{i}} \sum_{t=1}^{i-1}\left(\sum_{j=\mu_{i}}^{\mu_{t}-1} \beta_{t j} T^{j-\mu_{i}} v_{t}\right) .
$$

Set

$$
z=w-\sum_{t=1}^{i-1}\left(\sum_{j=\mu_{i}}^{\mu_{t}-1} \beta_{t j} T^{j-\mu_{i}} v_{t}\right) .
$$

It is easy to see that

$$
h\left(z, \mathcal{M}_{i-1}\right)=h\left(w, \mathcal{M}_{i-1}\right)=\nu_{i}, \quad h\left(z, \mathcal{M}+\mathcal{M}_{i-1}\right)=h\left(w, \mathcal{M}+\mathcal{M}_{i-1}\right)=\mu_{i}
$$

and $T^{\mu_{i}} z=\sum_{j=1}^{r} \alpha_{j} m_{j} \in \mathcal{M}$. Consequently, $T^{\nu_{i}} z=\sum_{j=1}^{r} \alpha_{j} T^{\nu_{i}-\mu_{i}} m_{j} \in \mathcal{M}_{i-1} \cap \mathcal{M}=$ $\vee\left\{m_{1}, \ldots, m_{s_{i-1}}\right\}$, so that $\alpha_{j}$ must be 0 if $j>s_{i-1}+\left(\nu_{i}-\mu_{i}\right)=s_{i}$. On the other hand, 
$h\left(z, \mathcal{M}_{i-1}\right)=\nu_{i}$ implies that $\alpha_{s_{i}} \neq 0$. Furthermore, since $T^{\mu_{i}}\left(\vee\left\{T z, \ldots, T^{s_{i}-1} z\right\}\right)=$ $\vee\left\{m_{1}, \ldots, m_{s_{i}-1}\right\}$, we can find a suitable $y \in \vee\left\{T z, \ldots, T^{s_{i}-1} z\right\}$ such that if $v_{i}=$ $\left(1 / \alpha_{s_{i}}\right) z-y$, we will have $T^{\mu_{i}} v_{i}=m_{s_{i}}$. Thus $v_{i}$ satisfy all the required conditions.

This finishes the induction and completes the proof.

Q.E.D.

Theorem 2.3. Let $T \in \mathcal{L}(\mathcal{H}), T^{\prime} \in \mathcal{L}\left(\mathcal{H}^{\prime}\right), \mathcal{M} \in \operatorname{Lat}(T)$, and $\mathcal{M}^{\prime} \in \operatorname{Lat}\left(T^{\prime}\right)$. Suppose that $T \sim T^{\prime}, T\left|\mathcal{M} \sim T^{\prime}\right| \mathcal{M}^{\prime}, \widehat{T}_{\mathcal{M}} \sim \widehat{T}^{\prime}{ }_{\mathcal{M}}^{\prime}$, and the operator $T \mid \mathcal{M}$ is cyclic. Then $(T, \mathcal{M}) \sim\left(T^{\prime}, \mathcal{M}^{\prime}\right)$.

Proof. Suppose $T$ is of the type $\nu$ and $\widehat{T}_{\mathcal{M}}$ is of the type $\mu$. Let $v_{1}, v_{2}, \ldots, v_{k}$ be the vectors constructed in the previous lemma, i.e., $\left\{m_{t}: 1 \leq t \leq\right.$ $r\} \cup\left\{T^{j} v_{i}: 1 \leq i \leq k, 0 \leq j \leq \mu_{i}-1\right\}$ is a basis of $\mathcal{H}$ and $T^{\mu_{i}}=m_{s_{i}}$ where $s_{i}=\sum_{t=1}^{i}\left(\nu_{t}-\mu_{t}\right)$, for $i=1, \ldots, k$. Let $m_{1}^{\prime}, \ldots, m_{r}^{\prime} \in \mathcal{M}^{\prime}$ and $v_{1}^{\prime}, \ldots, v_{k}^{\prime} \in \mathcal{H}^{\prime}$ be vectors with analogous properties with respect to $T^{\prime}$ and $\mathcal{M}^{\prime}$. Define $V: \mathcal{H} \rightarrow \mathcal{H}^{\prime}$ by $V m_{t}=m_{t}^{\prime}$ and $V T^{j} v_{i}=T^{\prime j} v_{i}^{\prime}$ for $1 \leq t \leq r, 1 \leq i \leq k$, and $0 \leq j \leq \mu_{i}-1$. Clearly $V$ induces a similarity between $(T, \mathcal{M})$ and $\left(T^{\prime}, \mathcal{M}^{\prime}\right)$.

Corollary 2.4. Let $T \in \mathcal{L}(\mathcal{H}), T^{\prime} \in \mathcal{L}\left(\mathcal{H}^{\prime}\right), \mathcal{M} \in \operatorname{Lat}(T)$, and $\mathcal{M}^{\prime} \in \operatorname{Lat}\left(T^{\prime}\right)$. Suppose that $T \sim T^{\prime}, T\left|\mathcal{M} \sim T^{\prime}\right| \mathcal{M}^{\prime}, \widehat{T}_{\mathcal{M}} \sim \widehat{T}_{\mathcal{M}^{\prime}}$, and the operator $\widehat{T}_{\mathcal{M}}: \mathcal{H} / \mathcal{M} \rightarrow \mathcal{H} / \mathcal{M}$ is cyclic. Then $(T, \mathcal{M}) \sim\left(T^{\prime} \mathcal{M}^{\prime}\right)$.

Proof. Let subspaces $\mathcal{L} \subset \mathcal{H}$ and $\mathcal{L}^{\prime} \subset \mathcal{H}^{\prime}$ be complementary to $\mathcal{M}$ and $\mathcal{M}^{\prime}$, respectively. Express $T$ and $T^{\prime}$ in the matrix form with respect to the decompositions $\mathcal{H}=\mathcal{M} \oplus \mathcal{L}$ and $\mathcal{H}^{\prime}=\mathcal{M}^{\prime} \oplus \mathcal{L}^{\prime}$. By passing to the transposes, we are in the situation of Theorem 2.3. We leave the details to the interested reader. Q.E.D.

Definition 2.5. An LR-pair is a pair of partitions $(\mu, \nu)$ satisfying

$$
\nu_{1} \geq \mu_{1} \geq \nu_{2} \geq \cdots \geq \nu_{i} \geq \mu_{i} \geq \nu_{i+1} \geq \cdots
$$

In terms of diagrams, $(\mu, \nu)$ is an LR-pair if and only if $\mu \subset \nu$ and every horizontal row of $\nu$ contains at most one point from $\nu \backslash \mu$, i.e., $0 \leq \bar{\nu}_{j}-\bar{\mu}_{j} \leq 1$.

Let $(\mu, \nu)$ be an LR-pair. Denote $r=|\nu|-|\mu|$ and let $\left\{j: \bar{\mu}_{j} \neq \bar{\nu}_{j}\right\}=$ $\left\{\delta_{1}, \delta_{2}, \ldots, \delta_{r}\right\}$ where $\delta_{1}>\delta_{2}>\cdots>\delta_{r}$. Denote by $\delta(\mu, \nu)$ the partition $\left(\delta_{1}, \delta_{2}, \ldots, \delta_{r}\right)$ and $\delta_{j}(\mu, \nu)=\delta_{j}$. Thus $\delta(\mu, \nu)$ marks all the row numbers where $\mu$ and $\nu$ are different. 
We shall illustrate LR-pairs via diagrams. The empty squares represent $\mu$, the empty squares along with the squares with 1 is the diagram of $\nu$.

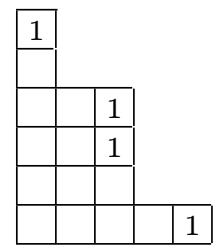

$$
\begin{aligned}
& \mu=(5,4,2,1) \quad \bar{\mu}=(4,3,2,2,1) \quad \delta(\mu, \nu)=(6,4,3,1) \\
& \nu=(6,4,4,1,1) \quad \bar{\nu}=(5,3,3,3,1,1)
\end{aligned}
$$

Figure 2.1

Let $T$ be an operator and $\mathcal{M} \in \operatorname{Lat}(T)$. Suppose that the quotient operator $\widehat{T}_{\mathcal{M}}$ is cyclic. Denote by $\mu$ and $\nu$ the types of $T \mid \mathcal{M}$ and $T$ respectively. Our goal now will be to characterize pairs of partitions which arise in this way.

Proposision 2.6. Let $(\mu, \nu)$ be an $L R$-pair and $\delta(\mu, \nu)=\left(\delta_{1}, \ldots, \delta_{r}\right)$ where $r=|\nu|-|\mu|$. Then there exist a vector space $\mathcal{K}, T \in \mathcal{L}(\mathcal{K}), \mathcal{M} \in \operatorname{Lat}(T)$, and $v \in \mathcal{N}\left(T^{\delta_{1}}\right)$, such that $T \mid \mathcal{M}$ is of type $\mu$, and, for each $j=1, \ldots, r$,

(1) $T^{j-1} v \in \mathcal{M}+\mathcal{N}\left(T^{\delta_{j}}\right)$,

(2) $T^{j-1} v \notin \mathcal{M}+\mathcal{N}\left(T^{\delta_{j}-1}\right)$,

(3) $T^{\delta_{j}+j-1} v \in T^{\delta_{j}} \mathcal{M}$,

(4) $T^{\delta_{j}+j-2} v \notin T^{\delta_{j}-1} \mathcal{M}$,

(5) $T^{r} v \in \mathcal{M}$.

Proof. Let $N$ be a large integer $(N>|\nu|)$ and let $\mathcal{K}$ be the vector space with a basis $\left\{e_{i j}: 1 \leq i, j \leq N\right\}$. Define $T \in \mathcal{L}(\mathcal{K})$ by $T e_{i j}=e_{i, j-1}$ if $j \geq 2$ and $T e_{i, 1}=0$. Further, define $T^{*} \in \mathcal{L}(\mathcal{K})$ by $T^{*} e_{i j}=e_{i, j+1}$ if $j<N$ and $T^{*} e_{i N}=0$. Let $\mathcal{M}=\vee\left\{e_{i j}: 1 \leq j \leq \mu_{i}\right\}$. Clearly $\mathcal{M} \in \operatorname{Lat}(T)$ and $T \mid \mathcal{M}$ is of type $\mu$.

We shall construct the vector $v \in \mathcal{K}$ by induction on $r$. For the trivial case $r=0$, we set $v=0$.

If $r=1$, let $\left\{\left(c, \delta_{1}\right)\right\}=\nu \backslash \mu$. Set $v=e_{c, \delta_{1}}$. Clearly $v \in \mathcal{N}\left(T^{\delta_{1}}\right)$, which implies (1) and (3). Further $v \notin \mathcal{M}+\mathcal{N}\left(T^{\delta_{1}-1}\right)$. Also, since $\left(c, \delta_{1}\right) \notin \mu$, we have $T^{\delta_{1}-1} e_{c, \delta_{1}}=e_{c, 1} \notin T^{\delta_{1}-1} \mathcal{M}$. Finally $T v=e_{c, \delta_{i}-1} \in \mathcal{M}$. Thus (1)-(5) are satisfied.

Suppose the statement is true for $r-1 \geq 1$. Let $\left(c, \delta_{1}\right) \in \nu \backslash \mu$. Obviously $\left(\mu, \nu \backslash\left(c, \delta_{1}\right)\right)$ is an LR-pair and $\delta\left(\mu, \nu \backslash\left(c, \delta_{1}\right)\right)=\left(\delta_{2}, \ldots, \delta_{r}\right)$. By the induction hypothesis, there exists $w \in \mathcal{N}\left(T^{\delta_{2}}\right)$ such that $T^{r-1} w \in \mathcal{M}$ and, for $j=1, \ldots, r-1$,

$$
T^{j-1} w \in \mathcal{M}+\mathcal{N}\left(T^{\delta_{j+1}}\right)
$$




$$
\begin{aligned}
& T^{j-1} w \notin \mathcal{M}+\mathcal{N}\left(T^{\delta_{j+1}-1}\right), \\
& T^{\delta_{j+1}+j-1} w \in T^{\delta_{j+1}} \mathcal{M}, \\
& T^{\delta_{j+1}+j-2} w \notin T^{\delta_{j+1}-1} \mathcal{M} .
\end{aligned}
$$

There are two cases we need to consider. If $\delta_{1}=\delta_{2}+1$, we set $v=T^{*} w$. If $\delta_{1}>\delta_{2}+1$, we set $v=T^{*} w+e_{c, \delta_{1}}$. In both cases $v \in \mathcal{N}\left(T^{\delta_{1}}\right)$ and $T e_{c, \delta_{1}} \in \mathcal{M}$. This and the induction assumption implies (5) and statements (1)-(4) for $j=2, \ldots, r$.

For $j=1,(1)$ and (3) follow from $v \in \mathcal{N}\left(T^{\delta_{1}}\right)$, and (4) implies (2). Indeed, if (2) is not true then $v \in \mathcal{M}+\mathcal{N}\left(T^{\delta_{1}-1}\right)$ so that $T^{\delta_{1}-1} v \in T^{\delta_{1}-1} \mathcal{M}$. Thus it is sufficient to show (4). If $\delta_{1}>\delta_{2}+1$ then $T^{\delta_{1}-1} v=T^{\delta_{1}-2} w+e_{c, 1}=e_{c, 1} \notin T^{\delta_{1}-1} \mathcal{M}$. If $\delta_{1}=\delta_{2}+1$ then $T^{\delta_{1}-1} v=T^{\delta_{2}-1} w \notin T^{\delta_{2}-1} \mathcal{M}$. But $T^{\delta_{1}-1} \mathcal{M} \subset T^{\delta_{2}-1} \mathcal{M}$, so that $T^{\delta_{1}-1} v \notin T^{\delta_{1}-1} \mathcal{M}$. This finishes the proof.

Q.E.D.

Lemma 2.7. Let $T \in \mathcal{L}(\mathcal{H})$ be of type $\nu, \mathcal{M} \in \operatorname{Lat}(T)$, and $T \mid \mathcal{M}$ be of type $\mu$. Suppose $v \in \mathcal{H}$ satisfies $\mathcal{H}=\mathcal{M} \vee\left\{v, T v, \ldots, T^{r-1} v\right\}$ and $T^{r} v \in \mathcal{M}$ where $r=|\nu|-|\mu|$. Denote $\delta_{j}=\min \left\{i: T^{j-1} v \in \mathcal{M}+\mathcal{N}\left(T^{i}\right)\right\}$ for each $j=1, \ldots, r$. Then $(\mu, \nu)$ is an LR-pair and $\left(\delta_{1}, \delta_{2}, \ldots, \delta_{r}\right)=\delta(\mu, \nu)$.

Proof. For each $j=1, \ldots, r$, we have $T^{j-1} v \in \mathcal{M}+\mathcal{N}\left(T^{\delta_{j}}\right)$ so that $T^{j} v \in \mathcal{M}+\mathcal{N}\left(T^{\delta_{j}-1}\right)$ and $\delta_{j+1} \leq \delta_{j}-1<\delta_{j}$. It is easy to see that $\mathcal{M}+\mathcal{N}\left(T^{k}\right)=$ $\mathcal{M} \vee\left\{T^{j-1} v: k \geq \delta_{j}\right\}$. Recall that $\operatorname{dim}\left(\mathcal{M} \cap \mathcal{N}\left(T^{k}\right)\right)-\operatorname{dim}\left(\mathcal{M} \cap \mathcal{N}\left(T^{k-1}\right)\right)=\bar{\mu}_{k} \quad$ (see Lemma 1.1). Since $\operatorname{dim}\left(\mathcal{M}+\mathcal{N}\left(T^{k}\right)\right)=\operatorname{dim} \mathcal{N}\left(T^{k}\right)+\operatorname{dim} \mathcal{M}-\operatorname{dim}\left(\mathcal{M} \cap \mathcal{N}\left(T^{k}\right)\right)$, we have

$$
\begin{aligned}
\bar{\nu}_{k} & =\operatorname{dim} \mathcal{N}\left(T^{k}\right)-\operatorname{dim} \mathcal{N}\left(T^{k-1}\right) \\
& =\operatorname{dim}\left(\mathcal{M} \cap \mathcal{N}\left(T^{k}\right)\right)-\operatorname{dim}\left(\mathcal{M} \cap \mathcal{N}\left(T^{k-1}\right)\right) \\
& +\operatorname{dim}\left(\mathcal{M}+\mathcal{N}\left(T^{k}\right)\right)-\operatorname{dim}\left(\mathcal{M}+\mathcal{N}\left(T^{k-1}\right)\right) \\
& =\bar{\mu}_{k}+\operatorname{card}\left\{j: \delta_{j} \leq k\right\}-\operatorname{card}\left\{j: \delta_{j} \leq k-1\right\} \\
& =\left\{\begin{array}{lll}
\bar{\mu}_{k}+1, & \text { if } \delta_{j}=k & \text { for some } j, \\
\bar{\mu}_{k}, & \text { if } \delta_{j} \neq k & \text { for all } j .
\end{array}\right.
\end{aligned}
$$

Thus, $(\mu, \nu)$ is an LR-pair and $\left(\delta_{1}, \delta_{2}, \ldots, \delta_{r}\right)=\delta(\mu, \nu)$.

Q.E.D.

Theorem 2.8. Let $\mu$ and $\nu$ be partitions. The following are equivalent.

(1) $(\mu, \nu)$ is an LR-pair.

(2) There exist $T \in \mathcal{L}(\mathcal{H})$ and $\mathcal{M} \in \operatorname{Lat}(T)$, such that $T \mid \mathcal{M}$ is of type $\mu, T$ is of type $\nu$, and $\widehat{T}_{\mathcal{M}}$ is cyclic of order $|\nu|-|\mu|$. 
Proof. The implication $(2) \Rightarrow(1)$ was proved in the previous lemma.

Let $(\mu, \nu)$ be an LR-pair and let $T \in \mathcal{L}(\mathcal{K}), \mathcal{M} \in \operatorname{Lat}(T)$ and $v \in \mathcal{K}$ satisfy the properties of Proposition 2.6. Set $\mathcal{H}=\mathcal{M} \vee\left\{T^{n} v: n \geq 0\right\}$ and denote by $\nu^{\prime}$ the type of $T \mid \mathcal{H}$. By Lemma 2.7, $\left(\mu, \nu^{\prime}\right)$ is an LR-pair and $\delta\left(\mu, \nu^{\prime}\right)=\left(\delta_{1}, \ldots, \delta_{r}\right)=\delta(\mu, \nu)$ so that $\nu^{\prime}=\nu$. Thus $T \mid \mathcal{H} \in \mathcal{L}(\mathcal{H})$ is the required operator.

Q.E.D.

The next result is a modification of Proposition 2.6.

Proposision 2.9. Let $(\mu, \nu)$ be an $L R$-pair and $\delta(\mu, \nu)=\left(\delta_{1}, \ldots, \delta_{r}\right)$ where $r=|\nu|$ $|\mu|$. Let $T \in \mathcal{L}(\mathcal{H})$ be of type $\nu, \mathcal{M} \in \operatorname{Lat}(T)$ and let $T \mid \mathcal{M}$ be of type $\mu$. Let $w \in \mathcal{H}$ satisfy $T^{r} w \in \mathcal{M}$ and $\mathcal{H}=\mathcal{M} \vee\left\{w, T w, \ldots, T^{r-1} w\right\}$. Then, for each $j=1, \ldots, r$,

(1) $T^{j-1} w \in \mathcal{M}+\mathcal{N}\left(T^{\delta_{j}}\right)$,

(2) $T^{j-1} w \notin \mathcal{M}+\mathcal{N}\left(T^{\delta_{j}-1}\right)$,

(3) $T^{\delta_{j}+j-1} w \in T^{\delta_{j}} \mathcal{M}$,

(4) $T^{\delta_{j}+j-2} w \notin T^{\delta_{j}-1} \mathcal{M}$,

(5) $\delta_{j}=\min \left\{k: h\left(w, T^{k} \mathcal{M}\right)<k+j\right\}$,

(6) $h\left(w, T^{\delta_{1}} \mathcal{M}\right)=\delta_{1}$ and $h\left(w, T^{k} \mathcal{M}\right)=k+j$ for $\delta_{j}>k \geq \delta_{j+1}, j=1, \ldots, r$ (as usually we set $\delta_{r+1}=0$ ).

Proof. By Corollary 2.4, $(T, \mathcal{M})$ is determined uniquely up to a similarity. Thus we may assume that $T \in \mathcal{L}(\mathcal{H})$ is the operator constructed in the previous theorem so that there exists $v \in \mathcal{H}$ satisfying $T^{r} v \in \mathcal{M}, \mathcal{H}=\mathcal{M} \vee\left\{v, T v, \ldots T^{r-1} v\right\}$ and properties (1)-(4) of Proposition 2.6 (which are identical with (1)-(4) of the present proposition with $w$ replaced by $v$ ).

The vector $w \in \mathcal{H}$ can be expressed as $w=m+\sum_{j=0}^{r-1} \alpha_{j} T^{j} v$ for some $m \in \mathcal{M}$, and $\alpha_{j} \in \mathbf{C}$. This implies (1) and (3). Similarly $v=m_{1}+\sum_{j=0}^{r-1} \beta_{j} T^{j} w$ for some $m_{1} \in \mathcal{M}$, and $\beta_{j} \in \mathbf{C}$ which implies (2) and (4).

For $j=1, \ldots, r$, properties (3) and (4) imply

$$
h\left(w, T^{\delta_{j}} \mathcal{M}\right)=h\left(w, T^{\delta_{j}-1} \mathcal{M}\right)=\delta_{j}+j-1 .
$$

Since $h\left(w, T^{k+1} \mathcal{M}\right) \leq h\left(w, T^{k} \mathcal{M}\right)+1$ for every $k$, the function $f(k)=h\left(w, T^{k} \mathcal{M}\right)-k$ is nonincreasing, $f\left(\delta_{j}\right)=j-1, f\left(\delta_{j}-1\right)=j$, and hence (5). Also $f(k)=j$ for $\delta_{j}>k \geq$ $\delta_{j+1}$ so that $h\left(w, T^{k} \mathcal{M}\right)=k+j$. For $j=r$ we have $f\left(\delta_{r+1}\right)=f(0)=r=f\left(\delta_{r}-1\right)$ so that (6) holds in this case too).

Q.E.D. 
Remark 2.10. $h\left(w, T^{k} \mathcal{M}\right)$ in the previous theorem is not determined for $k>\delta_{1}$. The vector $v$ costructed in Proposition 2.6 satisfies $v \in \mathcal{N}\left(T^{\delta_{1}}\right)$. Thus if $w=m+$ $\sum_{j=0}^{r-1} \alpha_{j} T^{j} v$ for some $m \in \mathcal{M}$ and $\alpha_{j} \in \mathbf{C}, \alpha_{0} \neq 0$ then $T^{i} w=T^{i} m$ for $i>\delta_{1}$, so that $h\left(w, T^{k} \mathcal{M}\right)=\max \left\{\delta_{1}, h\left(m, T^{k} \mathcal{M}\right)\right\}$.

\section{ADDING TWO BLOCKS AND MORE}

Definition 3.1. An LR-triple is a triple of partitions $(\mu, \nu, \rho)$ such that

(i) $(\mu, \nu)$ and $(\nu, \rho)$ are LR-pairs,

(ii) $\delta(\mu, \nu) \subset \delta(\nu, \rho)$.

We shall illustrate an LR-triple via a diagram. As before, the empty squares represent $\mu$, the empty squares along with the squares with 1 (resp. 1 and 2) is the diagram of $\nu$ (resp. $\rho$ ). Condition (ii) in Definition 3.1 means that "there are at least as many 1's as 2's above each horizontal line". In particular, if $(\mu, \nu, \rho)$ is an LR-triple then $\nu_{1}=\rho_{1}$. The following is an example of an LR-triple.

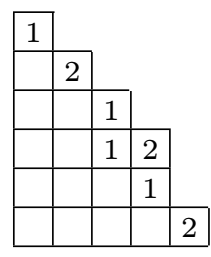

$$
\begin{array}{lll}
\mu=(5,4,2,1) & \bar{\mu}=(4,3,2,2,1) & \delta(\mu, \nu)=(6,4,3,2) \\
\nu=(6,4,4,2) & \bar{\nu}=(4,4,3,3,1,1) & \delta(\nu, \rho)=(5,3,1) \\
\rho=(6,5,4,3,1) & \bar{\rho}=(5,4,4,3,2,1) &
\end{array}
$$

Figure 3.1

The following lemma gives a characterization of LR-triples.

Lemma 3.2. Let $\mu, \nu$, and $\rho$ be partitions. Then $(\mu, \nu, \rho)$ is an LR-triple if and only if $(\mu, \nu)$ and $(\nu, \rho)$ are LR-pairs and

$$
\sum_{i=1}^{k+1}\left(\rho_{i}-\nu_{i}\right) \leq \sum_{i=1}^{k}\left(\nu_{i}-\mu_{i}\right)
$$

for all $k \geq 0$.

Proof. Let $(\mu, \nu, \rho)$ be an LR-triple and $k \geq 1$. Denote $\delta(\mu, \nu)=$ $\left(\delta_{1}, \ldots, \delta_{r}\right)$ and $\delta(\nu, \rho)=\left(\epsilon_{1}, \ldots, \epsilon_{p}\right)$ with $r=|\nu|-|\mu|$ and $p=|\rho|-|\nu|$. Then

$$
\sum_{i=1}^{k+1}\left(\rho_{i}-\nu_{i}\right)=\operatorname{card}\left\{j: \epsilon_{j}>\nu_{k+1}\right\} \leq \operatorname{card}\left\{j: \delta_{j}>\nu_{k+1}\right\} .
$$


If $\delta_{j}>\nu_{k+1}$ then $\left(c, \delta_{j}\right) \in \nu \backslash \mu$ for some $c \leq k$. Consequently $\delta_{j}>\mu_{k}$ and

$$
\sum_{i=1}^{k+1}\left(\rho_{i}-\nu_{i}\right) \leq \operatorname{card}\left\{j: \delta_{j}>\mu_{k}\right\}=\sum_{i=1}^{k}\left(\nu_{i}-\mu_{i}\right)
$$

for all $k \geq 1$. The inequality for $k=0$ follows from the fact that $\rho_{1}=\nu_{1}$.

Conversely, suppose

$$
\sum_{i=1}^{k+1}\left(\rho_{i}-\nu_{i}\right) \leq \sum_{i=1}^{k}\left(\nu_{i}-\mu_{i}\right)
$$

for all $k \geq 0$. Let $s \in \mathbf{N}$. If $s>\nu_{1}$, then $\operatorname{card}\left\{j: \epsilon_{j}>s\right\}=0$ since $\nu_{1}=\rho_{1}$. Now let $\nu_{k} \geq s>\nu_{k+1}$ for some $k \geq 1$. Observe that

$$
\operatorname{card}\left\{j: \epsilon_{j}>s\right\}=\sum_{i=1}^{k}\left(\rho_{i}-\nu_{i}\right)+\max \left\{\rho_{k+1}-s, 0\right\} .
$$

Here we have two cases to consider. If $\mu_{k}>s$ then

$$
\operatorname{card}\left\{j: \delta_{j}>s\right\}=\sum_{i=1}^{k}\left(\nu_{i}-\mu_{i}\right) \geq \sum_{i=1}^{k+1}\left(\rho_{i}-\nu_{i}\right) \geq \operatorname{card}\left\{j: \epsilon_{j}>s\right\} .
$$

Otherwise, if $\mu_{k} \leq s$, then

$$
\begin{aligned}
\operatorname{card}\left\{j: \delta_{j}>s\right\} & =\sum_{i=1}^{k-1}\left(\nu_{i}-\mu_{i}\right)+\left(\nu_{k}-s\right) \\
& \geq \sum_{i=1}^{k}\left(\rho_{i}-\nu_{i}\right)+\max \left\{\rho_{k+1}-s, 0\right\}=\operatorname{card}\left\{j: \epsilon_{j}>s\right\} .
\end{aligned}
$$

Hence, $\delta(\nu, \rho) \subset \delta(\mu, \nu)$.

Q.E.D.

Theorem 3.3. Let $(\mu, \nu, \rho)$ be an LR-triple, $T \in \mathcal{L}(\mathcal{H}), \mathcal{M}, \mathcal{L} \in \operatorname{Lat}(T)$, and $\mathcal{M} \subset \mathcal{L}$. Suppose $T|\mathcal{M}, T| \mathcal{L}$, and $T$ are of types $\mu, \nu$, and $\rho$ respectively, and that the operators $\widehat{T}_{\mathcal{L}}: \mathcal{H} / \mathcal{L} \rightarrow \mathcal{H} / \mathcal{L}$ and $\widehat{T}: \mathcal{L} / \mathcal{M} \rightarrow \mathcal{L} / \mathcal{M}$ induced by $T$ are cyclic. Denote $p=$ $|\rho|-|\nu|$. Then there exists $z \in \mathcal{H}$ such that $\mathcal{H}=\mathcal{L} \vee\left\{z, T z, \ldots, T^{p-1} z\right\}$ and $T^{p} z \in \mathcal{M}$. Furthermore, if we denote $\tilde{\mathcal{L}}=\mathcal{M} \vee\left\{z, T z, \ldots, T^{p-1} z\right\}$ and $\tilde{\nu}$ is the type of $T \mid \tilde{\mathcal{L}}$, then $\delta(\mu, \tilde{\nu}) \subset \delta(\mu, \nu)$.

Proof. Denote $r=|\nu|-|\mu|, \delta(\mu, \nu)=\left(\delta_{1}, \ldots, \delta_{r}\right)$, and $\delta(\nu, \rho)=$ $\left(\epsilon_{1}, \ldots, \epsilon_{p}\right)$. By Theorem 2.8 and Proposition 2.9, there exist vectors $v, w \in \mathcal{H}$ such 
that $\mathcal{L}=\mathcal{M} \vee\left\{v, T v, \ldots, T^{r-1} v\right\}, T^{r} v \in \mathcal{M}, \mathcal{H}=\mathcal{L} \vee\left\{w, T w, \ldots, T^{p-1} w\right\}, T^{p} w \in \mathcal{L}$ and

$$
\begin{array}{cc}
T^{j-1} w \in \mathcal{L}+\mathcal{N}\left(T^{\epsilon_{j}}\right), & j=1, \ldots, p, \\
T^{j-1} v \in \mathcal{M}+\mathcal{N}\left(T^{\delta_{j}}\right) \text { and } T^{j-1} v \notin \mathcal{M}+\mathcal{N}\left(T^{\delta_{j}-1}\right), & j=1, \ldots, r .
\end{array}
$$

Clearly also

$$
T^{j-1} v \notin \mathcal{M} \vee \mathcal{N}\left(T^{\delta_{j}-1}\right) \vee\left\{T^{j} v, T^{j+1} v, \ldots, T^{r-1} v\right\}
$$

since $T^{k} v \in \mathcal{M}+\mathcal{N}\left(T^{\delta_{k+1}}\right) \subset \mathcal{M}+\mathcal{N}\left(T^{\delta_{j}-1}\right)$ for $k \geq j$.

Claim. $T^{j} w \in \mathcal{M}+T^{c} \mathcal{L}+\mathcal{N}\left(T^{\epsilon_{j+1}}\right)$ for all $0 \leq c \leq j \leq p$. (Here we use the convention that $\left.\epsilon_{p+1}=0\right)$.

We prove the Claim by induction on $c$. For $c=0$, the Claim reduces to $T^{j} w \in \mathcal{L}+\mathcal{N}\left(T^{\epsilon_{j+1}}\right)$. Suppose the Claim holds for $0 \leq c-1 \leq j \leq p$. We prove it for $c$.

Fix $j$ satisfying $c \leq j \leq p$. By the induction assumption we can express

$$
T^{j} w=m+\sum_{k=c-1}^{r-1} \alpha_{k} T^{k} v+z
$$

for some $m \in \mathcal{M}, \alpha_{k} \in \mathbf{C}$, and $z \in \mathcal{N}\left(T^{\epsilon_{j+1}}\right)$. To complete the induction step, it suffices to show that $\alpha_{c-1}=0$. Suppose on the contrary $\alpha_{c-1} \neq 0$.

Since $c-1 \leq j-1 \leq p$, by the induction assumption we have also $T^{j-1} w \in \mathcal{M}+T^{c-1} \mathcal{L}+\mathcal{N}\left(T^{\epsilon_{j}}\right)$ so that $T^{j} w \in \mathcal{M}+T^{c} \mathcal{L}+\mathcal{N}\left(T^{\epsilon_{j}-1}\right)$. Together with (3.1) and the inequalities $\epsilon_{j}-1 \leq \epsilon_{c}-1 \leq \delta_{c}-1$ this gives

$$
\begin{aligned}
T^{c-1} v & \in\left\{T^{j} w\right\} \vee \mathcal{M} \vee\left\{T^{c} v, \ldots, T^{r-1} v\right\} \vee \mathcal{N}\left(T^{\epsilon_{j+1}}\right) \\
& \subset \mathcal{M} \vee\left\{T^{c} v, \ldots, T^{r-1} v\right\} \vee \mathcal{N}\left(T^{\epsilon_{j}-1}\right) \\
& \subset \mathcal{M} \vee\left\{T^{c} v, \ldots, T^{r-1} v\right\} \vee \mathcal{N}\left(T^{\delta_{c}-1}\right),
\end{aligned}
$$

a contradiction. Hence $\alpha_{c-1}=0$ and $T^{j} w \in \mathcal{M}+T^{c} \mathcal{L}+\mathcal{N}\left(T^{\epsilon_{j+1}}\right)$. This proves the Claim.

In particular, for $j=c=p$, we have $T^{p} w \in \mathcal{M}+T^{p} \mathcal{L}$. Write $T^{p} w=$ $m+T^{p} l$ for some $m \in \mathcal{M}$ and $l \in \mathcal{L}$. Set $z=w-l$. Then $T^{p} z=m \in M$ and $\mathcal{L} \vee\left\{z, T z, \ldots, T^{p-1} z\right\}=\mathcal{L} \vee\left\{w, T w, \ldots, T^{p-1} w\right\}=\mathcal{H}$

Let $\tilde{\mathcal{L}}=\mathcal{M} \vee\left\{z, T z, \ldots, T^{p} z\right\}$ and let $\tilde{\nu}$ be the type of $T \mid \tilde{\mathcal{L}}$. We observe that $T^{k} z \in \mathcal{M}+\mathcal{N}\left(T^{\delta_{k+1}}\right) \quad(k=0,1, \ldots)$. Indeed, by the Claim for $c=j=k$, we 
have $T^{k} z=T^{k} w-T^{k} l \in \mathcal{M}+T^{k} \mathcal{L}+\mathcal{N}\left(T^{\epsilon_{k+1}}\right) \subset \mathcal{M}+\mathcal{N}\left(T^{\delta_{k+1}}\right)$. Lemma 2.7 implies now $\delta(\mu, \tilde{\nu}) \subset \delta(\mu, \nu)$.

Q.E.D.

Remark 3.4. The previous theorem means that if $(\mu, \nu, \rho)$ is an LR-triple then one can construct $T \in \mathcal{L}(\mathcal{H}), \mathcal{M}, \mathcal{L} \in \operatorname{Lat}(T)$, such that $\mathcal{M} \subset \mathcal{L}$, operators $T|\mathcal{M}, T| \mathcal{L}$ and $T$ have types $\mu, \nu, \rho$ and the operator $\widehat{T}_{\mathcal{M}}: \mathcal{H} / \mathcal{M} \rightarrow \mathcal{H} / \mathcal{M}$ has the Jordan model consisting of two blocks of dimensions $|\nu|-|\mu|$ and $|\rho|-|\nu|$. Indeed, using Theorem 2.8 twice, we can obtain spaces $\mathcal{M} \subset \mathcal{L} \subset \mathcal{H}$ and an operator $T \in \mathcal{L}(\mathcal{H})$ satisfying the conditions of the previous theorem.

We generalize this result to n-tuples of partitions.

Definition 3.5. An LR-sequence is a sequence $\mu^{(0)}, \mu^{(1)}, \ldots$ of partitions with the property that $\left(\mu^{(i)}, \mu^{(i+1)}, \mu^{(i+2)}\right)$ is an LR-triple for every $i \geq 0$.

We identify a finite sequence $\mu^{(0)}, \mu^{(1)}, \ldots, \mu^{(n)}$ of partitions with an infinite sequence $\mu^{(0)}, \ldots, \mu^{(n)}, \mu^{(n)}, \mu^{(n)}, \ldots$.

Theorem 3.6. Let $\mu^{(0)}, \mu^{(1)}, \ldots, \mu^{(n)}$ be an LR-sequence. Then there exist an operator $T \in \mathcal{L}(\mathcal{H})$ and invariant subspaces $\mathcal{M}_{0} \subset \mathcal{M}_{1} \subset \ldots \subset \mathcal{M}_{n}=\mathcal{H}$, such that each $T \mid \mathcal{M}_{i}$ is of the type $\mu^{(i)}$ and the operator $\widehat{T}_{\mathcal{M}_{0}}: \mathcal{H} / \mathcal{M}_{0} \rightarrow \mathcal{H} / \mathcal{M}_{0}$ is of the type $\left(\left|\mu^{(1)}\right|-\left|\mu^{(0)}\right|,\left|\mu^{(2)}\right|-\left|\mu^{(1)}\right|, \ldots,\left|\mu^{(n)}\right|-\left|\mu^{(n-1)}\right|\right)$.

Proof. We proceed the proof by induction on $n$. The case $n=2$ is shown in the previous remark.

Suppose the statement is true for $n-1 \geq 2$. Let $\mathcal{M}_{0} \subset \mathcal{M}_{1} \subset \ldots \mathcal{M}_{n-1}$ and $T \in \mathcal{L}\left(\mathcal{M}_{n-1}\right)$ satisfy the conditions of the theorem. Using Theorem 2.8, we can find a space $\mathcal{H}=\mathcal{M}_{n} \supset \mathcal{M}_{n-1}$, a vector $v \in \mathcal{H}$, and an extension of $T$ to $\mathcal{H}$ such that $\mathcal{H}=\mathcal{M}_{n-1} \vee\left\{v, T v, \ldots, T^{r-1} v\right\}$ where $r=\left|\mu^{(n)}\right|-\left|\mu^{(n-1)}\right|=\operatorname{dim}\left(\mathcal{H} / \mathcal{M}_{n-1}\right)$, $T^{r} v \in \mathcal{M}_{n-1}$, and finally, $T \in \mathcal{L}(\mathcal{H})$ is of type $\mu^{(n)}$. The only problem is that $T^{r} v$ is an element of $\mathcal{M}_{n-1}$ and not necessarily of $\mathcal{M}_{0}$ as desired.

We prove the following statement, which will allow us to put $T^{r} v$ into $\mathcal{M}_{0}$, by the downward induction:

(3.2) For each $0 \leq s \leq n-1$ there exists $v_{s} \in \mathcal{H}$, such that $\mathcal{H}=\mathcal{M}_{n-1} \vee\left\{T^{n} v_{s}\right.$ : $n \geq 0\}, T^{r} v_{s} \in \mathcal{M}_{s}$ and $\delta\left(\mu^{(s)}, \tilde{\mu}^{(s+1)}\right) \subset \delta\left(\mu^{(s)}, \mu^{(s+1)}\right)$, where $\tilde{\mu}^{(s+1)}$ is the type of $T \mid \widetilde{\mathcal{M}}_{s+1}$, and $\widetilde{\mathcal{M}}_{s+1}=\mathcal{M}_{s} \vee\left\{v_{s}, T v_{s}, \ldots, T^{r-1} v_{s}\right\}$. 
Clearly (3.2) is true for $s=n-1$. Suppose it is true for $s \geq 1$. We prove that $\left(\mu^{(s-1)}, \mu^{(s)}, \tilde{\mu}^{(s+1)}\right)$ is an LR-triple. Indeed, $\left(\mu^{(s)}, \tilde{\mu}^{(s+1)}\right)$ is an LR-pair by Lemma 2.7 and, by the induction assumption,

$$
\delta\left(\mu^{(s)}, \tilde{\mu}^{(s+1)}\right) \subset \delta\left(\mu^{(s)}, \mu^{(s+1)}\right) \subset \delta\left(\mu^{(s-1)}, \mu^{(s)}\right) .
$$

By Theorem 3.3 for spaces $\mathcal{M}_{s-1} \subset \mathcal{M}_{s} \subset \tilde{\mathcal{M}}_{s+1}$, we can find a vector $v_{s-1} \in \tilde{\mathcal{M}}_{s+1} \subset \mathcal{H}$ such that $T^{r} v_{s-1} \in \mathcal{M}_{s-1}$ and $\mathcal{M}_{s} \vee\left\{v_{s-1}, T v_{s-1}, \ldots, T^{r-1} v_{s-1}\right\}=$ $\tilde{\mathcal{M}}_{s+1}$ so that

$$
\mathcal{M}_{n-1} \vee\left\{v_{s-1}, T v_{s-1}, \ldots, T^{r-1} v_{s-1}\right\} \supset \mathcal{M}_{n-1}+\tilde{\mathcal{M}}_{s+1}=\mathcal{H}
$$

Further, by Theorem 3.3, $\delta\left(\mu^{(s-1)}, \tilde{\mu}^{(s)}\right) \subset \delta\left(\mu^{(s-1)}, \mu^{(s)}\right)$ where $\tilde{\mu}^{(s)}$ is the type of $T \mid \widetilde{\mathcal{M}}_{s}$ and $\widetilde{\mathcal{M}}_{s}=\mathcal{M}_{s-1} \vee\left\{v_{s-1}, T v_{s-1}, \ldots, T^{r-1} v_{s-1}\right\}$.

Hence (3.2) is true for $s-1$. By induction we can obtain (3.2) for $s=0$ which finishes the proof.

Q.E.D.

Definition 3.7. Let $T \in \mathcal{L}(\mathcal{H})$ and $\mathcal{M} \in \operatorname{Lat}(T)$. Let $\left\{v_{1}, v_{2}, \ldots\right\}$ be a sequence of vectors of $\mathcal{H}$. Denote by $\mathcal{M}_{i}$ the smallest invariant subspace of $T$ containing $\left\{v_{1}, v_{2}, \ldots, v_{i}\right\}$. (We set $\left.\mathcal{M}_{0}=\{0\}\right)$. The sequence $\left\{v_{1}, v_{2}, \ldots\right\}$ is called maximal for $(T, \mathcal{M})$ if it satisfies

$$
h\left(v_{i}, \mathcal{M}_{i-1}+T^{k} \mathcal{M}\right)=\max _{x \in \mathcal{H}} h\left(x, \mathcal{M}_{i-1}+T^{k} \mathcal{M}\right)
$$

for every $k \geq 0$.

If $\left\{v_{1}, v_{2}, \ldots\right\}$ is a maximal sequence for $(T, \mathcal{M})$, then, for every $k=$ $0,1, \ldots$, the sequence $\left(h\left(v_{i}, \mathcal{M}_{i-1}+T^{k} \mathcal{M}\right)\right)_{i=1}^{\infty}$ is the type of the operator $\widehat{T}_{T^{k} \mathcal{M}}$ : $\mathcal{H} / T^{k} \mathcal{M} \rightarrow \mathcal{H} / T^{k} \mathcal{M}$ induced by $T$. Therefore the numbers $h\left(v_{i}, \mathcal{M}_{i-1}+T^{k} \mathcal{M}\right)$ do not depend on the choice of the maximal sequence $\left\{v_{1}, v_{2}, \ldots\right\}$. We denote by $g_{T, \mathcal{M}}(i, k)=$ $h\left(v_{i}, \mathcal{M}_{i-1}+T^{k} \mathcal{M}\right)$. We write $g(i, k)$ instead of $g_{T, \mathcal{M}}(i, k)$ if no ambiguity can arise. Clearly $g(i, 0) \leq g(i, 1) \leq g(i, 2) \leq \cdots$ and $g(1, k) \geq g(2, k) \geq \cdots$ for all $i, k$.

Proposition 3.8. Let $T \in \mathcal{L}(\mathcal{H}), \mathcal{M} \in \operatorname{Lat}(T)$ and let $\left\{v_{i}\right\}$ be a maximal sequence for $(T, \mathcal{M})$. Denote $\mathcal{M}_{0}=\{0\}$ and $\mathcal{M}_{i}=\vee\left\{T^{n} v_{t}: 1 \leq t \leq i, n \geq 0\right\}$. Let $\mu^{(i)}$ be the type of $T \mid\left(\mathcal{M}+\mathcal{M}_{i}\right)$. Then, for all $i, k \geq 0$,

(1) if $x \in \mathcal{H}, l \geq 0$ and $T^{l} x \in T^{k} \mathcal{M}+\mathcal{M}_{i}$ then $T^{l} x \in T^{k} \mathcal{M}+T^{l} \mathcal{M}_{i}$, 
(2) $\max \{k, g(i+1, k)\} \geq h\left(v_{i+1}, T^{k}\left(\mathcal{M}+\mathcal{M}_{i}\right)\right) \geq g(i+1, k)$,

(3)

$$
g(i+1, k)= \begin{cases}h\left(v_{i+1}, T^{k}\left(\mathcal{M} \vee \mathcal{M}_{i}\right)\right) & \text { if } k<\delta_{1}\left(\mu^{(i)}, \mu^{(i+1)}\right), \\ k & \text { if } \delta_{1}\left(\mu^{(i)}, \mu^{(i+1)}\right) \leq k \leq s, \\ s & \text { if } k \geq s,\end{cases}
$$

where $s=\min \left\{k: T^{k} \mathcal{M} \subset \mathcal{M}_{i}\right\}$,

(4) $\operatorname{dim}\left(T^{k} \mathcal{M} \cap \mathcal{M}_{i+1}\right)-\operatorname{dim}\left(T^{k} \mathcal{M} \cap \mathcal{M}_{i}\right)=g(i+1, K)-g(i+1, k)$ where

$K$ is a positive integer satisfying $T^{K} \mathcal{M}=\{0\}$,

(5) for $j \geq 1$,

$$
\delta_{j}\left(\mu^{(i)}, \mu^{(i+1)}\right)=\min \{k: g(i+1, k)<k+j\} .
$$

Proof. (1) For $i=1$ the statement is clear. Suppose the statement is true for $i-1 \geq 0$ and let $T^{l} x \in T^{k} \mathcal{M}+\mathcal{M}_{i}$. Then we can write $T^{l} x=z+\sum_{j=0}^{s-1} \alpha_{j} T^{j} v_{i}$ where $z \in T^{k} \mathcal{M}+\mathcal{M}_{i-1}, \alpha_{j} \in \mathbf{C}$ and $s=h\left(v_{i}, T^{k} \mathcal{M}+\mathcal{M}_{i-1}\right)$. If $\alpha_{j}=0$ for every $j<s$ then $T^{l} x \in T^{k} \mathcal{M}+\mathcal{M}_{i-1}$ so that we can use the induction assumption. Let $s_{0}=\min \left\{j: \alpha_{j} \neq 0\right\}<s$. Then

$$
h\left(x, T^{k} \mathcal{M}+\mathcal{M}_{i-1}\right)=l+s-s_{0} \leq s=h\left(v_{i}, T^{k} \mathcal{M}+\mathcal{M}_{i-1}\right) .
$$

Thus $s_{0} \geq l$. Set $y=x-\sum_{j=s_{0}}^{s-1} \alpha_{j} T^{j-l} v_{i}$ so that $T^{l} y=z \in T^{k} \mathcal{M}+\mathcal{M}_{i-1}$. By the induction assumption $T^{l} y \in T^{k} \mathcal{M}+T^{l} \mathcal{M}_{i-1}$ so that $T^{l} x \in T^{k} \mathcal{M}+T^{l} \mathcal{M}_{i}$.

(2) Fix $i, k$. The second inequality is clear because $T^{k}\left(\mathcal{M}+\mathcal{M}_{i}\right) \subset$ $T^{k} \mathcal{M}+\mathcal{M}_{i}$. Let $l=\max \{k, g(i+1, k)\}$. Then $T^{l} v_{i+1} \in T^{k} \mathcal{M}+\mathcal{M}_{i}$ so that $T^{l} v_{i+1} \in$ $T^{k} \mathcal{M}+T^{l} \mathcal{M}_{i}$. Thus $h\left(v_{i+1}, T^{k}\left(\mathcal{M}+\mathcal{M}_{i}\right)\right) \leq l$.

(3) If $k<\delta_{1}\left(\mu^{(i)}, \mu^{(i+1)}\right)=\delta_{1}$, then, by Proposition $2.9, h\left(v_{i+1}, T^{k}(\mathcal{M}+\right.$ $\left.\left.\mathcal{M}_{i}\right)\right)>k$ so that $g(i+1, k)=h\left(v_{i+1}, T^{k}\left(\mathcal{M}+\mathcal{M}_{i}\right)\right)$ by $(2)$.

Further $T^{\delta_{1}} v_{i+1} \in T^{\delta_{1}}\left(\mathcal{M}+\mathcal{M}_{i}\right)$ so that, for $k \geq \delta_{1}, T^{k} v_{i+1} \in T^{k}(\mathcal{M}+$ $\left.\mathcal{M}_{i}\right)$ and

$$
\begin{aligned}
g(i+1, k) & =h\left(v_{i+1}, T^{k} \mathcal{M}+\mathcal{M}_{i}\right)=\max \left\{h\left(x, T^{k} \mathcal{M}+\mathcal{M}_{i}\right): x \in \mathcal{H}\right\} \\
& =\max \left\{h\left(x, T^{k} \mathcal{M}+\mathcal{M}_{i}\right): x \in \mathcal{M}+\mathcal{M}_{i}\right\}
\end{aligned}
$$

If we consider the operator $\widehat{T}:\left(\mathcal{M}+\mathcal{M}_{i}\right) / \mathcal{M}_{i} \rightarrow\left(\mathcal{M}+\mathcal{M}_{i}\right) / \mathcal{M}_{i}$ induced by $T$ and denote $s=\min \left\{k: \widehat{T}^{k}=0\right\}=\min \left\{k: T^{k} \mathcal{M} \subset \mathcal{M}_{i}\right\}$ then

$$
\max \left\{h\left(x, T^{k} \mathcal{M}+\mathcal{M}_{i}\right): x \in \mathcal{M}+\mathcal{M}_{i}\right\}= \begin{cases}k & \text { if }(k \leq s), \\ s & \text { if }(k \geq s),\end{cases}
$$


so that (3) holds.

(4) We have

$$
T^{k} \mathcal{M}+\mathcal{M}_{i+1}=\left(T^{k} \mathcal{M}+\mathcal{M}_{i}\right) \vee\left\{T^{j} v_{i+1}: 0 \leq j<h\left(v_{i+1}, T^{k} \mathcal{M}+\mathcal{M}_{i}\right)\right\}
$$

so that

$$
\operatorname{dim}\left(T^{k} \mathcal{M}+\mathcal{M}_{i+1}\right)-\operatorname{dim}\left(T^{k} \mathcal{M}+\mathcal{M}_{i}\right)=h\left(v_{i+1}, T^{k} \mathcal{M}+\mathcal{M}_{i}\right)=g(i+1, k) .
$$

Similarly

$$
\operatorname{dim} \mathcal{M}_{i+1}-\operatorname{dim} \mathcal{M}_{i}=g(i+1, K),
$$

where $K$ is a positive integer big enough $\left(T^{K} \mathcal{M}=\{0\}\right)$. Thus

$$
\begin{aligned}
& \operatorname{dim}\left(T^{k} \mathcal{M} \cap \mathcal{M}_{i+1}\right)-\operatorname{dim}\left(T^{k} \mathcal{M} \cap \mathcal{M}_{i}\right) \\
= & \operatorname{dim} T^{k} \mathcal{M}+\operatorname{dim} \mathcal{M}_{i+1}-\operatorname{dim}\left(T^{k} \mathcal{M}+\mathcal{M}_{i+1}\right) \\
& \quad-\operatorname{dim} T^{k} \mathcal{M}-\operatorname{dim} \mathcal{M}_{i}+\operatorname{dim}\left(T^{k} \mathcal{M}+\mathcal{M}_{i}\right) \\
& =g(i+1, K)-g(i+1, k) .
\end{aligned}
$$

(5) Since, by Proposition 2.9 (5),

$$
\delta_{j}\left(\mu^{(i)}, \mu^{(i+1)}\right)=\min \left\{k: h\left(v_{i+1}, T^{k}\left(\mathcal{M}+\mathcal{M}_{i}\right)\right)<k+j\right\}
$$

it is sufficient to show that

$$
h\left(v_{i+1}, T^{k}\left(\mathcal{M}+\mathcal{M}_{i}\right)\right)<k+j \Longleftrightarrow g(i+1, k)<k+j .
$$

The implication " $\Rightarrow$ " follows from $h\left(v_{i+1}, T^{k}\left(\mathcal{M}+\mathcal{M}_{i}\right)\right) \geq g(i+1, k)$. If $h\left(v_{i+1}, T^{k}(\mathcal{M}+\right.$ $\left.\left.\mathcal{M}_{i}\right)\right) \geq k+j$ then, by $(2), h\left(v_{i+1}, T^{k}\left(\mathcal{M}+\mathcal{M}_{i}\right)\right)=g(i+1, k)$.

Q.E.D.

Theorem 3.9. The sequence $\left(\mu^{(i)}\right)$ of partitions defined in the previous proposition is an $L R$-sequence which does not depend on the choice of a maximal sequence $\left\{v_{j}\right\}$.

Proof. Clearly $\left(\mu^{(i)}, \mu^{(i+1)}\right)$ is an LR-pair by Lemma 2.7. Further, by Proposition $3.8(5)$ and inequality $g(i+2, k) \leq g(i+1, k)$ we have $\delta\left(\mu^{(i+1)}, \mu^{(i+2)}\right) \subset$ $\delta\left(\mu^{(i)}, \mu^{(i+1)}\right)$ so that $\left(\mu^{(i)}, \mu^{(i+1)}, \mu^{(i+2)}\right)$ is an LR-triple.

Clearly $\mu^{(0)}$ is the type of $T \mid \mathcal{M}$ so that it does not depend on $\left\{v_{j}\right\}$. 
Suppose that $\mu^{(i-1)}$ does not depend on the choice of $\left\{v_{j}\right\}$ for some $i-1 \geq$ 0 . Since the function $g$ does not depend on the choice of $\left\{v_{j}\right\}$, statement (5) of the previous proposition implies that $\mu^{(i)}$ is indepentent of $\left\{v_{j}\right\}$.

Q.E.D.

Definition 3.10. Let $T \in \mathcal{L}(\mathcal{H}), \mathcal{M} \in \operatorname{Lat}(T)$, and let $\left\{v_{j}\right\}$ be a maximal sequence for $(T, \mathcal{M})$. Let $\mu^{(i)}$ be the type of $T \mid\left(\mathcal{M}+\mathcal{M}_{i}\right)$, where $\mathcal{M}_{i}=\vee\left\{T^{n} v_{t}: 1 \leq t \leq i, n \geq 0\right\}$. Then the sequence $\left(\mu^{(i)}\right)$ is called the $L R$-sequence of $(T, \mathcal{M})$.

Clearly $\mu^{(i)}$ is constant for $i$ big enough.

Remark 3.11. There is another standard way to assign an LR-sequence to a pair $(T, \mathcal{M})$ which was intensively studied, see [6]. For each $k \geq 0$ denote by $\lambda^{(k)}$ the type of the operator $\widehat{T}_{T^{k} \mathcal{M}}: \mathcal{H} / T^{k} \mathcal{M} \rightarrow \mathcal{H} / T^{k} \mathcal{M}$. Then the conjugated partitions $\overline{\lambda^{(0)}}, \overline{\lambda^{(1)}}, \ldots, \overline{\lambda^{(n)}}$ where $n$ is the order of $T \mid \mathcal{M}$ form an LR-sequence (in the sense of Definition 3.5). This sequence is different from the sequence $\left(\mu^{(i)}\right)$ defined above (even the numbers of partitions do not agree). However, sequences $\left(\lambda^{(k)}\right)$ and $\left(\mu^{(i)}\right)$ determine each other via Proposition 3.8 (4) and (5).

Theorem 3.12. Let $T \in \mathcal{L}(\mathcal{H}), T^{\prime} \in \mathcal{L}\left(\mathcal{H}^{\prime}\right), \mathcal{M} \in \operatorname{Lat}(T), \mathcal{M}^{\prime} \in \operatorname{Lat}\left(T^{\prime}\right)$. The following statements are equivalent:

(1) $g_{T, \mathcal{M}}=g_{T^{\prime}, \mathcal{M}^{\prime}}$,

(2) operators $\widehat{T}_{T^{k} \mathcal{M}}: \mathcal{H} / T^{k} \mathcal{M} \rightarrow \mathcal{H} / T^{k} \mathcal{M}$ and $\widehat{T}_{T^{\prime k} \mathcal{M}^{\prime}}^{\prime}: \mathcal{H}^{\prime} / T^{\prime k} \mathcal{M}^{\prime} \rightarrow$ $\mathcal{H}^{\prime} / T^{\prime}{ }^{k} \mathcal{M}^{\prime}$ are similar for every $k=0,1, \ldots$,

(3) $(T, \mathcal{M})$ and $\left(T^{\prime}, \mathcal{M}^{\prime}\right)$ have the same LR-sequences.

Proof. Clearly (1) and (2) are equivalent. The implication (1) $\Rightarrow(3)$ follows from Proposition 3.8 (5).

To show $(3) \Rightarrow(1)$, suppose the LR-sequence $\left(\mu^{(i)}\right)$ of $(T, \mathcal{M})$ is given.

Fix $k \geq 0$. Let $\left\{v_{i}\right\}$ be a maximal sequence for $(T, \mathcal{M})$ and let $\mathcal{M}_{i}=$ $\vee\left\{T^{n} v_{t}: 1 \leq t \leq i, n \geq 0\right\}$. We prove by induction on $j$ that the LR-sequence $\left(\mu^{(i)}\right)$ determines the following quantities:

(a) $\operatorname{dim}\left(T^{k} \mathcal{M} \cap \mathcal{M}_{j}\right)$,

(b) $g(j+1, k)$.

Clearly (a) is determined for $j=0$. By Propositions 3.8 (3) and 2.9 (6), (a) determines (b) for every $j$. On the other hand $g(j+1, k)$ determine $\operatorname{dim}\left(T^{k} \mathcal{M} \cap\right.$ 
$\left.\mathcal{M}_{j+1}\right)$ by Proposition $3.8(4)$. Thus $g(j+1, k)$ is determined for all $j, k \geq 0$, and hence $(3) \Rightarrow(1)$.

\section{ADMISSIBLE TRIPLES}

For each $T \in \mathcal{L}(\mathcal{H})$ and $\mathcal{M} \in \operatorname{Lat}(T)$, one can associate a triple $(\alpha, \beta, \gamma)$ such that the type of $T \mid \mathcal{M}$ is $\alpha$, of $\widehat{T}_{\mathcal{M}}$ is $\beta$, and of $T$ is $\gamma$. In this section will study the structure of all the triples $(\alpha, \beta, \gamma)$ that arise in this way.

Definition 4.1. A triple $(\alpha, \beta, \gamma)$ of partitions is called admissible if there exist an operator $T$ and $\mathcal{M} \in \operatorname{Lat}(T)$ such that $T \mid \mathcal{M}, \widehat{T}_{\mathcal{M}}$, and $T$, are of types $\alpha, \beta$, and $\gamma$, respectively.

Fix $n \geq 1$. We restrict our attention in this section to operators $T$ of multiplicity $\leq n$. In other words the type of $T$ is a partition $\gamma=\left(\gamma_{1}, \ldots, \gamma_{n}\right)$. If $\mathcal{M} \in \operatorname{Lat}(T)$ then the same is true for operators $T \mid \mathcal{M}$ and $\widehat{T}_{\mathcal{M}}$.

Theorems 3.6 and 3.9 give a characterization of admissible triples in terms of LR-sequences: a triple $(\alpha, \beta, \gamma)$ is admissible if and only if there exists an LR-sequence $\left(\mu^{(0)}, \mu^{(1)}, \ldots, \mu^{(n)}\right)$ such that $\alpha=\mu^{(0)}, \beta=\left(\left|\mu^{(1)}\right|-\left|\mu^{(0)}\right|,\left|\mu^{(2)}\right|-\left|\mu^{(1)}\right|, \ldots,\left|\mu^{(n)}\right|-\right.$ $\left.\left|\mu^{(n-1)}\right|\right)$, and $\gamma=\mu^{(n)}$.

One can generalize the notion of partitions to real numbers. A generalized partition is a sequence of nonincreasing nonnegative numbers. If $a=\left(a_{1}, a_{2}, \ldots, a_{n}\right)$ and $b=\left(b_{1}, b_{2}, \ldots, b_{n}\right)$ are generalized partitions, we say $a \subset b$ if and only if $a_{i} \leq b_{i}$ for every $i$. It is obvious that the set of all generalized partitions is a cone in $\mathbf{R}^{(n)}$ with the usual addition and multiplication by scalars on $\mathbf{R}^{(n)}$, and these operations coincide with the usual definitions of addition and multiplication by integers for partitions.

For the rest of this section, we will freely exchange the notations $c=$ $\left(c_{j}^{(i)}\right) \in \mathbf{R}^{n(n+1)}$ and $c=\left(c^{(0)}, c^{(1)}, \cdots, c^{(n)}\right) \in \mathbf{R}^{n} \times \mathbf{R}^{n} \times \cdots \times \mathbf{R}^{n}$, where $c^{(i)}=$ $\left(c_{1}^{(i)}, c_{2}^{(i)}, \ldots, c_{n}^{(i)}\right)$. Let $C$ be the set of all points $c=\left(c_{j}^{(i)}\right)$ of $\mathbf{R}^{n(n+1)}$ such that, for all $i, j$,

$$
c_{j-1}^{(i-1)} \geq c_{j}^{(i)} \geq c_{j}^{(i-1)}
$$

and

$$
\sum_{k=1}^{j-1}\left(c_{k}^{(i)}-c_{k}^{(i-1)}\right) \geq \sum_{k=1}^{j}\left(c_{k}^{(i+1)}-c_{k}^{(i)}\right) .
$$


Condition (1) implies that $c^{(i)}=\left(c_{1}^{(i)}, c_{2}^{(i)}, \ldots, c_{n}^{(i)}\right)$ are generalized partitions for each $i$ and $c^{(0)} \subset c^{(1)} \subset \cdots \subset c^{(n)}$.

Clearly LR-sequences are precisely the points of $C$ with integer coordinates.

Lemma 4.2. $C$ is a cone in $\mathbf{R}^{n(n+1)}$, i.e., if $x, y \in C, r \geq 0$, then $x+y \in C$, and $r x \in C$.

When $C$ is restricted to points of integer coordinates, we have the following useful fact about LR-sequences.

\section{Corollary 4.3.}

(i) If $\left(\lambda^{(0)}, \lambda^{(1)}, \ldots, \lambda^{(n)}\right)$ and $\left(\mu^{(0)}, \mu^{(1)}, \ldots, \mu^{(n)}\right)$ are LR-sequences then $\left(\lambda^{(0)}+\mu^{(0)}, \lambda^{(1)}+\mu^{(1)}, \ldots, \lambda^{(n)}+\mu^{(n)}\right)$ is an LR-sequence.

(2) Ifk $\in \mathbf{N}$ and $\left(\lambda^{(0)}, \ldots, \lambda^{(n)}\right)$ is a sequence of partitions then $\left(\lambda^{(0)}, \ldots, \lambda^{(n)}\right)$ is an LR-sequence if and only if $\left(k \lambda^{(0)}, \ldots, k \lambda^{(n)}\right)$ is an LR-sequence.

Denote $P=\left\{\left(x_{j}^{(i)}\right): x_{j}^{(i)} \geq 0\right.$ for all $0 \leq i \leq n, 1 \leq j \leq n$ and $\sum_{i, j} x_{j}^{(i)} \leq$ 1\}. Clearly $C=\bigcup_{r \geq 0} r(P \cap C)$ and $P \cap C$ is a compact polyhedral set (i.e., $P \cap C$ is determined by a finite number of linear inequalities). Denote by $E$ the set of all vertices (i.e., extremal points) of $P \cap C$. It is well-known that $P \cap C$ coincide with conv $(E)$, the convex hull of $E$ and the set $E$ is finite. Clearly all points of $E$ have rational coordinates since they are intersections of hyperplanes with rational coefficients.

An admissible triple $(\alpha, \beta, \gamma)$ can be regarded as the point

$$
\left(\alpha_{1}, \alpha_{2}, \ldots, \alpha_{n}, \beta_{1}, \beta_{2}, \ldots, \beta_{n}, \gamma_{1}, \gamma_{2}, \ldots, \gamma_{n}\right) \in \mathbf{R}^{3 n}
$$

Define the linear mapping $\Psi: \mathbf{R}^{n(n+1)} \rightarrow \mathbf{R}^{3 n}$ by

$$
\begin{aligned}
\Psi\left(\left(c_{j}^{(i)}\right)\right)=( & c_{1}^{(0)}, c_{2}^{(0)}, \ldots, c_{n}^{(0)}, \sum_{j=1}^{n}\left(c_{j}^{(1)}-c_{j}^{(0)}\right), \sum_{j=1}^{n}\left(c_{j}^{(2)}-c_{j}^{(1)}\right), \ldots, \\
& \left.\sum_{j=1}^{n}\left(c_{j}^{(n)}-c_{j}^{(n-1)}\right), c_{1}^{(n)}, c_{2}^{(n)}, \ldots, c_{n}^{(n)}\right) .
\end{aligned}
$$

We summarize some of the properties of $C$ and $\Psi$ in the following statement. 


\section{Proposition 4.4.}

(1) $\Psi(C)=\bigcup_{r \geq 0} r \cdot \Psi(P \cap C)$,

(2) $\Psi(P \cap C)=\operatorname{conv}\{\Psi e, e \in E\}$ so that $\Psi(P \cap C)$ is a polytop,

(3) all points $\{\Psi e: e \in E\}$ have rational coordinates,

(4) a triple $x=(\alpha, \beta, \gamma) \in \mathbf{R}^{3 n}$ is admissible if and only if $x=\Psi y$ for some $y \in C \cap \mathbf{Z}^{n(n+1)}$.

Together with Corollary 4.3, it is easy to see that the set of all the admissible triples is a "cone".

Corollary 4.5. If $(\alpha, \beta, \gamma)$ and $\left(\alpha^{\prime}, \beta^{\prime}, \gamma^{\prime}\right)$ are admissible triples then $\left(\alpha+\alpha^{\prime}, \beta+\right.$ $\left.\beta^{\prime}, \gamma+\gamma^{\prime}\right)$ is admissible. If $k \in \mathbf{N}$ and $(\alpha, \beta, \gamma)$ is a triple of partitions then $(\alpha, \beta, \gamma)$ is admissible if and only if $(k \alpha, k \beta, k \gamma)$ is admissible.

Theorem 4.6. A triple of partitions $(\alpha, \beta, \gamma)$ is admissible if and only if it lies in $\Psi(C)$.

Proof. Clearly admissible triples lie in $\Psi(C)$. Conversely, let $(\alpha, \beta, \gamma) \in$ $\Psi(C) \cap \mathbf{Z}^{3 n}$. Then $(\alpha, \beta, \gamma)=\sum_{e \in E} t_{e} \cdot(\Psi e)$ for some rational coefficients $t_{e}$. For each $e \in E$ there exists a positive integer $r_{e}$ such that $r_{e} \cdot e \in \mathbf{Z}^{n(n+1)}$, so that $\Psi\left(r_{e} \cdot e\right)$ is admissible. Thus $(\alpha, \beta, \gamma)=\sum_{e \in E} \frac{t_{e}}{r_{e}} \Psi\left(r_{e} \cdot e\right)$. Let $q$ be the common denominator of $\frac{t_{e}}{r_{e}}$, that is for all $e \in E, \frac{t_{e}}{r_{e}}=\frac{p_{e}}{q}$ for some integers $p_{e}$. Then $q \cdot(\alpha, \beta, \gamma)=\sum_{e \in E} p_{e} \cdot \Psi\left(r_{e} \cdot e\right)$ so that $q \cdot(\alpha, \beta, \gamma)$ is admissible and so is $(\alpha, \beta, \gamma)$.

Q.E.D.

Corollary 4.7. There exists a finite set of admissible triples $\left\{\left(\alpha^{(s)}, \beta^{(s)}, \gamma^{(s)}\right)\right\}_{s}$ such that a triple of partitions $(\alpha, \beta, \gamma)$ is admissible if and only if $(\alpha, \beta, \gamma)=\sum_{s} a_{s}$. $\left(\alpha^{(s)}, \beta^{(s)}, \gamma^{(s)}\right)$ for some rational coefficients $a_{s}$.

Proof. It is sufficient to take the set $\left\{\Psi\left(r_{e} \cdot e\right), e \in E\right\}$ from the previous proof.

Q.E.D.

Corollary 4.8. For each $n \geq 1$, there exist a finite number of linear inequalities with integer coefficients such that a triple of partitions of length at most $n$ is admissible if and only if it satisfies all of them.

Proof. The set $\Psi(P \cap C)$ is a polytop so it is defined by a finite number of hyperplanes (see [9]). The hyperplanes have rational coefficients since the vertices of 
$\Psi(P \cap C)$ have rational coordinates. Since $\Psi(C)$ is a cone, $\Psi(C)=\bigcup_{r \geq 0} r \cdot \Psi(P \cap C)$, $\Psi(C)$ is bounded by those hyperplanes which pass through the origin. Since an equation of a hyperplane passing through the origin can be multiplied by a suitable integer to obtain integer coefficients, the proof is finished.

Q.E.D.

Remark 4.9. (i) Explicit form of inequalities determining admissible triples for $n \leq 4$ was obtained in [12].

(ii) In [4], Gohberg and Kaashoek raised the question whether it is possible to characterize addmissible triples by hyperplanes. Corollary 4.8 gives an affirmative answer. The considerations of this section show in principle, how these hyperplanes can be obtained, however, it would be interesting to know these hyperplanes explicitly.

\section{EQUIVALENCES OF INVARIANT SUPSPACES}

In this section we study equivalence relations between invariant subspaces.

Definition 5.1. Let $T$ and $T^{\prime}$ be operators on $\mathcal{H}$ and $\mathcal{H}^{\prime}, \Gamma$ and $\Gamma^{\prime}$ be sublattices of $\operatorname{Lat}(T)$ and $\operatorname{Lat}\left(T^{\prime}\right)$ respectively. Suppose that $\mathcal{L} \in \Gamma$ implies $T \mathcal{L}, T^{-1} \mathcal{L} \in \Gamma$. We say $\Gamma$ and $\Gamma^{\prime}$ are $\left(T, T^{\prime}\right)$-(lattice) isomorphic if there exists a surjective mapping $\phi: \Gamma \rightarrow \Gamma^{\prime}$ such that, for every $\mathcal{L}, \mathcal{L}_{1} \in \Gamma$,

(i) $\phi\left(\mathcal{L}+\mathcal{L}_{1}\right)=\phi(\mathcal{L})+\phi\left(\mathcal{L}_{1}\right)$,

(ii) $\phi\left(\mathcal{L} \cap \mathcal{L}_{1}\right)=\phi(\mathcal{L}) \cap \phi\left(\mathcal{L}_{1}\right)$,

(iii) $\phi(T \mathcal{L})=T^{\prime} \phi(\mathcal{L})$

(iv) $\phi\left(T^{-1}(\mathcal{L})\right)=T^{\prime-1} \phi(\mathcal{L})$,

(v) $\operatorname{dim} \phi(\mathcal{L})=\operatorname{dim} \mathcal{L}$.

Clearly a $\left(T, T^{\prime}\right)$ isomorphism is one-to-one and $T^{\prime} \mathcal{L}^{\prime}, T^{-1} \mathcal{L}^{\prime} \in \Gamma^{\prime}$ if $\mathcal{L}^{\prime} \in$ $\Gamma$. If $T$ and $T^{\prime}$ are similar, then it is easy to see that the similarity induces a $\left(T, T^{\prime}\right)$ isomorphism between $\operatorname{Lat}(T)$ and $\operatorname{Lat}\left(T^{\prime}\right)$.

Let $\mathcal{M}$ be an invariant subspace of $T \in \mathcal{L}(\mathcal{H})$. Denote by $\mathcal{L}_{T, \mathcal{M}}$ the smallest lattice of invariant subspaces of $T$ such that

(i) $\mathcal{M} \in \mathcal{L}_{T, \mathcal{M}}$, and

(ii) if $\mathcal{L} \in \mathcal{L}_{T, \mathcal{M}}$, then $T \mathcal{L} \in \mathcal{L}_{T, \mathcal{M}}$ and $T^{-1} \mathcal{L} \in \mathcal{L}_{T, \mathcal{M}}$. 
Recall that if $\mathcal{M} \in \operatorname{Lat}(T)$ and $\mathcal{M}^{\prime} \in \operatorname{Lat}\left(T^{\prime}\right)$, we say that $(T, \mathcal{M}) \sim$ $\left(T^{\prime}, \mathcal{M}^{\prime}\right)$ if and only if there exists an invertible operator $V: \mathcal{H} \rightarrow \mathcal{H}^{\prime}$ such that $V T=T^{\prime} V$ and $V \mathcal{M}=\mathcal{M}^{\prime}$. We will define a weaker equivalence relation on the collection of $(T, \mathcal{M})$ via lattice isomorphisms.

Definition 5.2. Let $T \in \mathcal{L}(\mathcal{H}), T^{\prime} \in \mathcal{L}\left(\mathcal{H}^{\prime}\right), \mathcal{M} \in \operatorname{Lat}(T)$, and $\mathcal{M}^{\prime} \in \operatorname{Lat}\left(T^{\prime}\right)$. Then $(T, \mathcal{M}) \stackrel{\operatorname{dim}}{\sim}\left(T^{\prime}, \mathcal{M}^{\prime}\right)$ if there exists a $\left(T, T^{\prime}\right)$-lattice isomorphism $\phi: \mathcal{L}_{T, \mathcal{M}} \rightarrow \mathcal{L}_{T^{\prime}, \mathcal{M}^{\prime}}$ such that $\phi(\mathcal{M})=\mathcal{M}^{\prime}$.

Clearly $(T, M) \sim\left(T^{\prime}, M^{\prime}\right)$ implies $(T, M) \stackrel{\operatorname{dim}}{\sim}\left(T^{\prime}, M^{\prime}\right)$.

It is usually rather difficult to describe the lattice $\mathcal{L}_{T, \mathcal{M}}$ (we give an example that it can be infinite). Therefore we introduce the following weaker equivalences which were already implicitly used in the previous sections.

Definition 5.3. Let $T \in \mathcal{L}(\mathcal{H}), T^{\prime} \in \mathcal{L}\left(\mathcal{H}^{\prime}\right), \mathcal{M} \in \operatorname{Lat}(T)$ and $\mathcal{M}^{\prime} \in \operatorname{Lat}\left(T^{\prime}\right)$. We write $(T, \mathcal{M}) \stackrel{\mathrm{LR}}{\sim}\left(T^{\prime}, \mathcal{M}^{\prime}\right)$ if $(T, \mathcal{M})$ and $\left(T^{\prime}, \mathcal{M}^{\prime}\right)$ have the same LR-sequences (see Theorem 3.12). We write $(T, \mathcal{M}) \stackrel{\mathrm{J}}{\sim}\left(T^{\prime}, \mathcal{M}^{\prime}\right)$ if $T \sim T^{\prime}, T\left|\mathcal{M} \sim T^{\prime}\right| \mathcal{M}^{\prime}$ and $\widehat{T}_{\mathcal{M}} \sim \widehat{T}_{\mathcal{M}^{\prime}}$.

Lemma 5.4. Let $T \in \mathcal{L}(\mathcal{H}), T^{\prime} \in \mathcal{L}\left(\mathcal{H}^{\prime}\right), \mathcal{M} \in \operatorname{Lat}(T)$ and $\mathcal{M}^{\prime} \in \operatorname{Lat}\left(T^{\prime}\right)$. The following are equivalent:

(1) $(T, \mathcal{M}) \sim\left(T^{\prime}, \mathcal{M}^{\prime}\right)$.

(2) $\operatorname{dim}\left(T^{i} \mathcal{H}+T^{j} \mathcal{M}\right)=\operatorname{dim}\left(T^{\prime i} \mathcal{H}+T^{\prime j} \mathcal{M}^{\prime}\right)$ for all $i, j \geq 0$

(3) $\operatorname{dim}\left(T^{i} \mathcal{H}\right)=\operatorname{dim}\left(T^{i} \mathcal{H}\right)$ and $\operatorname{dim}\left(T^{i} \mathcal{H} \cap T^{j} \mathcal{M}\right)=\operatorname{dim}\left(T^{\prime i} \mathcal{H} \cap T^{\prime j} \mathcal{M}^{\prime}\right)$ for all $i, j \geq 0$.

Proof. It is easy to see that (2) and (3) are equivalent. By Theorem 3.12, $(T, \mathcal{M}) \stackrel{\text { LR }}{\sim}\left(T^{\prime}, \mathcal{M}^{\prime}\right)$ if and only if $\widehat{T}_{T^{j} \mathcal{M}} \sim \widehat{T}_{T^{\prime j} \mathcal{M}^{\prime}}^{\prime}$ for every $j \geq 0$. The Jordan model of $\widehat{T}_{T^{j} \mathcal{M}}: \mathcal{H} / T^{j} \mathcal{M} \rightarrow \mathcal{H} / T^{j} \mathcal{M}$ is determined by the dimensions of the ranges

$$
\begin{aligned}
& \operatorname{dim}\left(\left(\widehat{T}_{T^{j} \mathcal{M}}\right)^{i}\left(\mathcal{H}+T^{j} \mathcal{M}\right)\right)=\operatorname{dim}\left(T^{i} \mathcal{H}+T^{j} \mathcal{M}\right)-\operatorname{dim}\left(T^{j} \mathcal{M}\right) \\
= & \operatorname{dim}\left(T^{i} \mathcal{H}\right)-\operatorname{dim}\left(T^{i} \mathcal{H} \cap T^{j} \mathcal{M}\right) .
\end{aligned}
$$

Q.E.D.

Our next theorem follows immediately from the definitions of each equivalence relation. 
Theorem 5.5. $(T, \mathcal{M}) \sim\left(T^{\prime}, \mathcal{M}^{\prime}\right) \Rightarrow(T, M) \stackrel{\operatorname{dim}}{\sim}\left(T^{\prime}, \mathcal{M}^{\prime}\right) \Rightarrow(T, \mathcal{M}) \stackrel{\text { LR }}{\sim}\left(T^{\prime}, \mathcal{M}^{\prime}\right) \Rightarrow$ $(T, \mathcal{M}) \stackrel{\mathrm{J}}{\sim}\left(T^{\prime}, \mathcal{M}^{\prime}\right)$

However none of the implications in the previous theorem can be reversed as the following examples illustrate. Recall that an operator $T \in \mathcal{L}(\mathcal{H})$ is said to be the standard operator of type $\left(\lambda_{1}, \lambda_{2}, \ldots\right)$, if $\mathcal{H}$ is the vector space with a basis $\left\{e_{i j}: 1 \leq j \leq \lambda_{i}\right\}$ and $T e_{i j}=e_{i, j-1}$ if $j \geq 2$ and $T e_{i 1}=0$.

Example 5.6. $(T, \mathcal{M}) \stackrel{\mathrm{J}}{\sim}\left(T, \mathcal{M}^{\prime}\right) \not \rightarrow(T, \mathcal{M}) \stackrel{\mathrm{LR}}{\sim}\left(T, \mathcal{M}^{\prime}\right)$.

Construction: Let $T$ be the standard operator of type $(3,2,1)$ and $\mathcal{M}=$ $\vee\left\{e_{11}, e_{21}, e_{22}\right\}$ and $\mathcal{M}^{\prime}=\vee\left\{e_{11}, e_{12}, e_{31}\right\}$, see Figure 5.1.

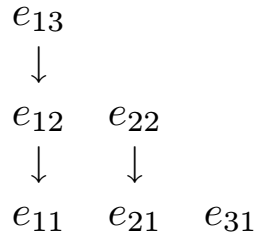

$(T, \mathcal{M})$

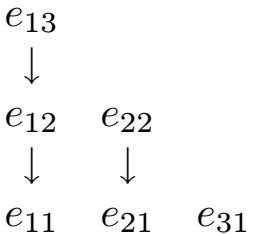

$\left(T, \mathcal{M}^{\prime}\right)$

Figure 5.1

Clearly $\mathcal{M}, \mathcal{M}^{\prime} \in \operatorname{Lat}(T), T|\mathcal{M} \sim T| \mathcal{M}^{\prime}$ since both restrictions are of type $(2,1)$, and operators $\widehat{T}_{\mathcal{M}}: \mathcal{H} / \mathcal{M} \rightarrow \mathcal{H} / \mathcal{M}$ and $\widehat{T}^{\prime}{ }_{\mathcal{M}^{\prime}}: \mathcal{H} / \mathcal{M}^{\prime} \rightarrow \mathcal{H} / \mathcal{M}^{\prime}$ are also similar since they are also of type $(2,1)$. Thus $(T, \mathcal{M}) \stackrel{\mathrm{J}}{\sim}\left(T, \mathcal{M}^{\prime}\right)$.

On the other hand, $(T, \mathcal{M})$ and $\left(T, \mathcal{M}^{\prime}\right)$ are not LR-equivalent since we have $\operatorname{dim}\left(T^{2} \mathcal{H} \cap T \mathcal{M}\right)=0$ and $\operatorname{dim}\left(T^{2} \mathcal{H} \cap T \mathcal{M}^{\prime}\right)=1$.

In some cases, however, $\stackrel{\mathrm{J}}{\sim}$ implies $\sim$.

Theorem 5.7. Let $(T, \mathcal{M}) \stackrel{J}{\sim}\left(T^{\prime}, \mathcal{M}^{\prime}\right)$ and let one of the following conditions be satisfied.

(1) $T$ is uniform,

(2) $T \mid \mathcal{M}$ is cyclic,

(3) $\widehat{T}_{\mathcal{M}}: \mathcal{H} / \mathcal{M} \rightarrow \mathcal{H} / \mathcal{M}$ is cyclic.

Then $(T, \mathcal{M}) \sim\left(T^{\prime}, \mathcal{M}^{\prime}\right)$.

Proof. (i) was proved in [3], (ii) and (iii) in Theorem 2.3 and Corollary 2.4 .

Q.E.D. 
However, as we will see in the next example, it is not possible to combine conditions (a) and (b) (or (a) and (c)) of the previous theorem.

Example 5.8. There exist pairs $(T, \mathcal{M}) \stackrel{\mathrm{J}}{\sim}\left(T, \mathcal{M}^{\prime}\right)$ which are not LR-equivalent and $\widehat{T}_{\mathcal{M}}: H / \mathcal{M} \rightarrow H / \mathcal{M}$ is uniform.

Construction: Let $T \in \mathcal{L}(\mathcal{H})$ be the standard operator of type $(4,3,2,1)$. Let $\mathcal{M}=\vee\left\{e_{11}, e_{12}, e_{21}, e_{22}, e_{23}, e_{41}\right\}$ and $\mathcal{M}^{\prime}=\vee\left\{e_{11}, e_{12}, e_{21}, e_{31}, e_{13}+e_{32}, e_{22}+e_{41}\right\}$, see Figure 5.2.

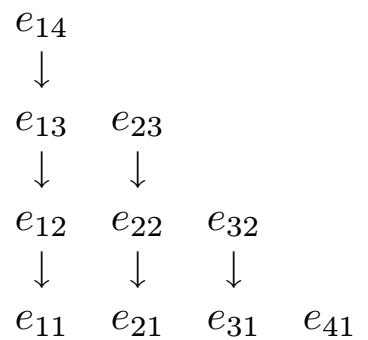

$(T, \mathcal{M})$

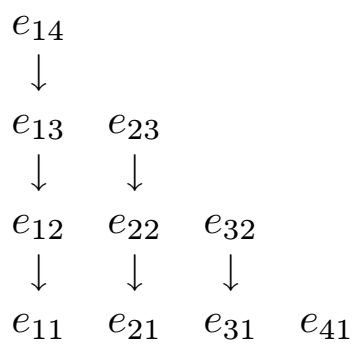

$\left(T, \mathcal{M}^{\prime}\right)$

Figure 5.2

Then $T \mid \mathcal{M}$ is of type $(3,2,1)$ and so is $T \mid \mathcal{M}^{\prime}$. Indeed, the Jordan model of $T \mid \mathcal{M}^{\prime}$ can be described as: $e_{13}+e_{32} \stackrel{T}{\longrightarrow} e_{12}+e_{31} \stackrel{T}{\longrightarrow} e_{11}, e_{22}+e_{41} \stackrel{T}{\longrightarrow} e_{21}, e_{31}$. So $T|\mathcal{M} \sim T| \mathcal{M}^{\prime}$

Similarly $\widehat{T}_{\mathcal{M}}: \mathcal{H} / \mathcal{M} \rightarrow \mathcal{H} / \mathcal{M}$ is of type $(2,2)$ and so is $\widehat{T}_{\mathcal{M}^{\prime}}^{\prime}: \mathcal{H} / \mathcal{M}^{\prime} \rightarrow$ $\mathcal{H} / \mathcal{M}^{\prime}\left(e_{14}+\mathcal{M}^{\prime} \rightarrow e_{13}+\mathcal{M}^{\prime} \rightarrow \mathcal{M}^{\prime}, e_{23}+\mathcal{M}^{\prime} \rightarrow e_{22}+\mathcal{M}^{\prime} \rightarrow \mathcal{M}^{\prime}\right)$. Thus $(T, \mathcal{M}) \stackrel{J}{\sim}$ $\left(T, \mathcal{M}^{\prime}\right)$ and $\widehat{T}_{\mathcal{M}}$ is uniform. Further $(T, \mathcal{M})$ and $\left(T, \mathcal{M}^{\prime}\right)$ are not LR-equivalent since $T^{3} \mathcal{H} \cap T^{2} \mathcal{M}=\{0\}$ and $T^{3} \mathcal{H} \cap T^{2} \mathcal{M}^{\prime}=\vee\left\{e_{11}\right\}$

Similar example can be done for uniform $T \mid \mathcal{M}$ (we can use duality as in Corollary 2.4.).

Example 5.9. $(T, \mathcal{M}) \stackrel{\operatorname{LR}}{\sim}\left(T, \mathcal{M}^{\prime}\right) \not \rightarrow(T, \mathcal{M}) \stackrel{\operatorname{dim}}{\sim}\left(T, \mathcal{M}^{\prime}\right)$.

Construction: Let $T$ be the standard operator of type $(5,4,2,1)$. Set $\mathcal{M}=\vee\left\{e_{11}, e_{21}, e_{12}+e_{31}, e_{22}+e_{41}\right\}$ and $\mathcal{M}^{\prime}=\vee\left\{e_{11}, e_{21}, e_{12}+e_{41}, e_{22}+e_{31}\right\}$, see Figure 5.3. 


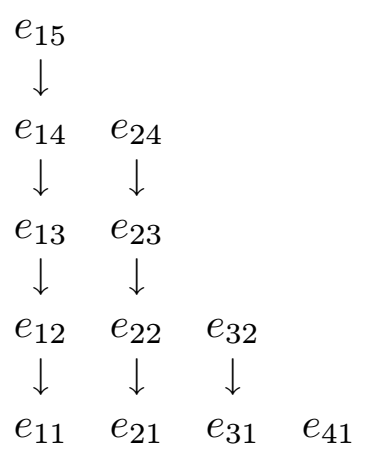

$(T, \mathcal{M})$

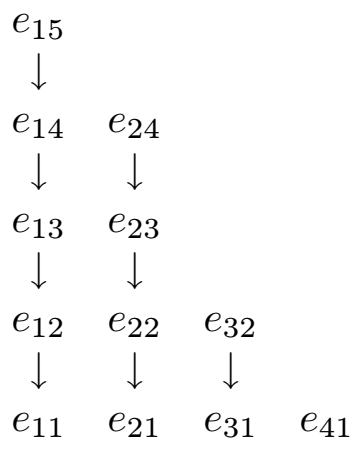

$\left(T, \mathcal{M}^{\prime}\right)$

Figure 5.3

Clearly $T \mathcal{M} \subset \mathcal{M}$ and $T \mathcal{M}^{\prime} \subset \mathcal{M}^{\prime}$. Then $(T, \mathcal{M})$ and $\left(T, \mathcal{M}^{\prime}\right)$ are not dim-equivalent since $T(T \mathcal{H} \cap \mathcal{M}) \cap T^{4} \mathcal{H}=\vee\left\{e_{11}\right\}$ and $T\left(T \mathcal{H} \cap \mathcal{M}^{\prime}\right) \cap T^{4} H=\{0\}$. We prove $(T, \mathcal{M}) \stackrel{\text { LR }}{\sim}\left(T, \mathcal{M}^{\prime}\right)$, i.e., $\operatorname{dim}\left(T^{i} \mathcal{H} \cap T^{j} \mathcal{M}\right)=\operatorname{dim}\left(T^{i} \mathcal{H} \cap T^{j} \mathcal{M}^{\prime}\right)$ for every $i, j \geq 0$. This is clear for $j \geq 2$ since then $T^{j} \mathcal{M}=T^{j} \mathcal{M}^{\prime}=\{0\}$. Also for $j=1$ we have $T \mathcal{M}=T \mathcal{M}^{\prime}=\vee\left\{e_{11}, e_{21}\right\}$. Let $j=0$. For $i=0,1, \ldots, 4$, we have

$$
\begin{aligned}
& \operatorname{dim}(H \cap \mathcal{M})=\operatorname{dim} \mathcal{M}=4=\operatorname{dim}\left(\mathcal{H} \cap \mathcal{M}^{\prime}\right), \\
& \operatorname{dim}(T \mathcal{H} \cap \mathcal{M})=3=\operatorname{dim}\left(T \mathcal{H} \cap \mathcal{M}^{\prime}\right), \\
& \operatorname{dim}\left(T^{2} \mathcal{H} \cap \mathcal{M}\right)=2=\operatorname{dim}\left(T^{2} \mathcal{H} \cap \mathcal{M}^{\prime}\right), \\
& \operatorname{dim}\left(T^{3} \mathcal{H} \cap \mathcal{M}\right)=2=\operatorname{dim}\left(T^{3} \mathcal{H} \cap \mathcal{M}^{\prime}\right), \\
& \operatorname{dim}\left(T^{4} \mathcal{H} \cap \mathcal{M}\right)=1=\operatorname{dim}\left(T^{4} \mathcal{H} \cap \mathcal{M}^{\prime}\right)
\end{aligned}
$$

For $i \geq 5$ we have $T^{i} \mathcal{H}=\{0\}$. Thus $(T, \mathcal{M}) \stackrel{\text { LR }}{\sim}\left(T, \mathcal{M}^{\prime}\right)$.

Example 5.10. $(T, \mathcal{M}) \stackrel{\operatorname{dim}}{\sim}\left(T, \mathcal{M}^{\prime}\right) \nRightarrow(T, \mathcal{M}) \sim\left(T, \mathcal{M}^{\prime}\right)$.

Construction: Let $T$ be the standard operator of type $(7,6,5,4,3)$. Let $q_{1}, q_{2}$ be two distinct transcendental numbers. For $i=1,2$, set

$$
\begin{aligned}
\mathcal{M}_{i}=\vee & \left\{e_{11}, e_{21}, e_{22}+e_{31}, e_{13}+e_{23}+e_{42}, e_{12}+e_{22}+e_{41},\right. \\
& \left.q_{i} e_{14}+e_{24}+e_{53}, q_{i} e_{13}+e_{23}+e_{52}, q_{i} e_{12}+e_{22}+e_{51}\right\}
\end{aligned}
$$

as indicated in the figure below. 


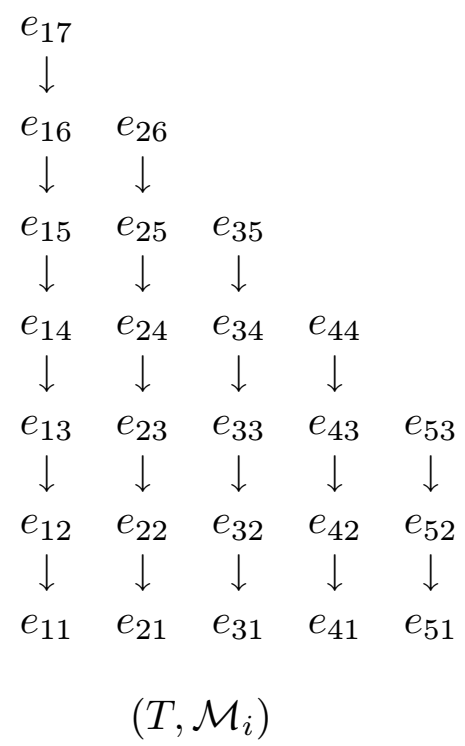

Figure 5.4

(a) We show first that $\left(T, \mathcal{M}_{1}\right) \not\left(T, \mathcal{M}_{2}\right)$. Note that, $T^{6} H=\vee\left\{e_{11}\right\}$, $T\left(\mathcal{M}_{i} \cap T^{4} \mathcal{H}\right)=\vee\left\{e_{21}\right\}, T^{2}\left(\mathcal{M}_{i} \cap T^{2} \mathcal{H}\right)=\vee\left\{e_{11}+e_{21}\right\}$, and $T^{3} \mathcal{M}_{i}=\vee\left\{q_{i} e_{11}+e_{21}\right\}$ for $i=1,2$.

Suppose $V: \mathcal{H} \rightarrow \mathcal{H}$ is an invertible operator satisfying $V T=T V$ and $V \mathcal{M}_{1}=\mathcal{M}_{2}$. Then $V\left(T^{6} \mathcal{H}\right)=T^{6} \mathcal{H}, V\left(T\left(\mathcal{M}_{1} \cap T^{4} \mathcal{H}\right)\right)=T\left(\mathcal{M}_{2} \cap T^{4} \mathcal{H}\right)$ so that $V e_{11}=$ $\alpha e_{11}, V e_{21}=\beta e_{21}$ for some $\alpha, \beta \in C$. Further $V\left(T^{2}\left(\mathcal{M}_{1} \cap T^{2} \mathcal{M}\right)\right)=T^{2}\left(\mathcal{M}_{2} \cap T^{2} \mathcal{M}\right)$, we must have $V\left(e_{11}+e_{21}\right)=\alpha e_{11}+\beta e_{21} \in \vee\left\{e_{11}+e_{21}\right\}$. Thus $\beta=\alpha$. Finally from $V\left(T^{3} \mathcal{M}_{1}\right)=T^{3} \mathcal{M}_{2}$ we deduce that $V\left(q_{1} e_{11}+e_{21}\right)$, must be a multiple of $q_{2} e_{21}+e_{21}$. But $V\left(q_{1} e_{11}+e_{21}\right)=\alpha q_{1} e_{11}+\alpha e_{21}$, which implies that $q_{1}=q_{2}$, a contradiction.

(b) We show now that $\left(T, \mathcal{M}_{1}\right) \stackrel{\operatorname{dim}}{\sim}\left(T, \mathcal{M}_{2}\right)$. Denote by $\mathbf{Q}$ the field of rational numbers and by $\mathbf{Q}_{i}=\mathbf{Q}\left[q_{i}\right]$ the field generated by the rational numbers and $q_{i}(i=1,2)$. Obviously $\mathbf{Q}_{1}$ and $\mathbf{Q}_{2}$ are isomorphic and the isomorphism $\psi: \mathbf{Q}_{1} \rightarrow \mathbf{Q}_{2}$ satisfies

$$
\psi\left(\sum_{j=-\infty}^{\infty} r_{j} q_{1}^{j}\right)=\sum_{j=-\infty}^{\infty} r_{j} q_{2}^{j}
$$

for all $r_{j} \in \mathbf{Q}\left(r_{j}=0\right.$ for all but a finitely many j's).

Consider vector spaces $\mathbf{Q}_{1}^{25}$ and $\mathbf{Q}_{2}^{25}$ over $\mathbf{Q}_{1}$ and $\mathbf{Q}_{2}$, respectively. Clearly $\psi$ extends naturally to an isomorphism $\psi^{25}: \mathbf{Q}_{1}^{25} \rightarrow \mathbf{Q}_{2}^{25}$ between these vector spaces. 
In particular, if $\mathcal{L}$ is a subspace of $\mathbf{Q}_{1}^{25}$ then $\psi^{25} \mathcal{L}$ is a subspace of $\mathbf{Q}_{2}^{25}$ and

$$
\operatorname{dim}_{\mathbf{Q}_{2}}\left(\psi^{25} \mathcal{L}\right)=\operatorname{dim}_{\mathbf{Q}_{1}}(\mathcal{L})
$$

where $\operatorname{dim}_{\mathbf{Q}_{i}}$ denotes the dimension over the field $\mathbf{Q}_{i}$. Identify now $\mathcal{H}$ with $\mathbf{C}^{25}$ using the basis $\left\{e_{i j}\right\}$. Let $\mathcal{Y}_{i}(i=1,2)$ be the set of subspaces $\mathcal{L}$ of $\mathcal{H}\left(=\mathbf{C}^{25}\right)$ such that $\operatorname{dim}_{\mathbf{C}} \mathcal{L}=\operatorname{dim}_{\mathbf{Q}_{i}}\left(\mathcal{L} \cap \mathbf{Q}_{i}^{25}\right)$. In other words, $\mathcal{L} \in \mathcal{Y}_{i}$ if and only if $\mathcal{L}$ has a basis consisting of elements from $\mathbf{Q}_{i}^{25}$. By standard linear algebra methods $\mathcal{L} \in \mathcal{Y}_{i}$ if and only if $\mathcal{L}$ is the set of all solutions of a finite number of linear equations with coefficients from $\mathbf{Q}_{i}$.

Clearly $\mathcal{M}_{1} \in \mathcal{Y}_{1}$ and $\mathcal{M}_{2} \in \mathcal{Y}_{2}$. Using the above characterization of $\mathcal{Y}_{1}$ and $\mathcal{Y}_{2}$ we show easily that $\mathcal{L}, \mathcal{L}^{\prime} \in Y_{i}$ implies $\mathcal{L}+\mathcal{L}^{\prime} \in Y_{i}, \mathcal{L} \cap \mathcal{L}^{\prime} \in Y_{i}, T \mathcal{L} \in Y_{i}$, $T^{-1} \mathcal{L} \in Y_{i}$. Thus $\mathcal{L}_{T, \mathcal{M}_{1}} \subset \mathcal{Y}_{1}, \mathcal{L}_{T, \mathcal{M}_{2}} \subset \mathcal{Y}_{2}$. For $\mathcal{L} \in \mathcal{Y}_{1}$ we have

$$
\operatorname{dim}_{\mathbf{C}} \mathcal{L}=\operatorname{dim}_{\mathbf{Q}_{1}}\left(\mathcal{L} \cap \mathbf{Q}_{1}^{25}\right)=\operatorname{dim}_{\mathbf{Q}_{2}} \psi^{25}\left(\mathcal{L} \cap \mathbf{Q}_{1}^{25}\right)
$$

Define $\phi: \mathcal{Y}_{1} \rightarrow \mathcal{Y}_{2}$ by $\phi(\mathcal{L})=\psi^{25}\left(\mathcal{L} \cap \mathbf{Q}_{1}^{25}\right)$. It is easy to see that $\phi$ is a $(\mathrm{T}, \mathrm{T})$-lattice isomorphism between $\mathcal{Y}_{1}$ and $\mathcal{Y}_{2}$ and $\phi\left(\mathcal{M}_{1}\right)=\mathcal{M}_{2}$. Hence the restriction of $\phi$ to $\mathcal{L}_{\mathcal{M}, T}$ is a $(\mathrm{T}, \mathrm{T})$-lattice isomorphism betweent $\mathcal{L}_{\mathcal{M}_{2}, T}$ and $\mathcal{L}_{\mathcal{M}_{2}, T}$. Thus we conclude that $\left(T, \mathcal{M}_{1}\right) \stackrel{\operatorname{dim}}{\sim}\left(T, \mathcal{M}_{2}\right)$.

It is easy to see that for the operator $T$ from the previous example there exist uncountably many subspaces $\mathcal{M}_{\alpha} \in \operatorname{Lat}(T)$ ( $\alpha$ transcendental) such that $\left(T, \mathcal{M}_{\alpha_{1}}\right) \nsim\left(T, \mathcal{M}_{\alpha_{2}}\right)$ if $\alpha_{1} \neq \alpha_{2}$. Thus the number of $\sim$-equivalence classes is continuum.

Obviously for given operator $T$ there are only finitely many non- $\stackrel{J}{\sim}$ equivalent or non- $\stackrel{L R}{\sim}$-equivalent subspaces $\mathcal{M} \in \operatorname{Lat}(T)$. We show that the number of

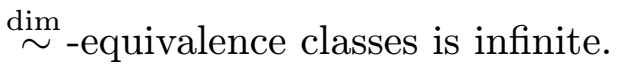

Lemma 5.11. Suppose $\Gamma$ is a lattice of subspaces of a vector space $\mathcal{H}$, let $a, b, c$ be linearly independent vectors in $\mathcal{H}$ such that $\vee\{a\}, \vee\{b\}, \vee\{c\}, \vee\{a+c\}$ and $\vee\{b+c\} \in \Gamma$. Then $\vee\{a-k b\} \in \Gamma \quad(k=0,1, \ldots)$, so that $\Gamma$ is infinite.

Proof. We prove the statement by induction on $k$. The statement is clear for $k=0$. Suppose $\vee\{a-k b\} \in \Gamma$. Then

$$
(\vee\{a-k b, c\}) \cap(\vee\{a+c, b\})=\vee\{a-k b+c\} \in \Gamma
$$


and

$$
(\vee\{a-k b+c, b+c\}) \cap(\vee\{a, b\})=\vee\{a-(k+1) b\} \in \Gamma .
$$

Thus $\vee\{a-k b\} \in \Gamma$ for every $k=0,1, \ldots$

Q.E.D.

\section{Example 5.12.}

(1) There exist an operator $T$ in a finite-dimensional space $\mathcal{H}$ and $\mathcal{M} \in$ $\operatorname{Lat}(T)$ such that the lattice $\mathcal{L}_{T, \mathcal{M}}$ is infinite.

(2) There exist subspaces $\mathcal{M}_{k} \in \operatorname{Lat}(T)(k=1,2, \ldots)$ such that the pairs $\left(T, \mathcal{M}_{k}\right)$ are mutually non $\stackrel{\operatorname{dim}}{\sim}$-equivalent.

Construction: Let $T \in \mathcal{L}(\mathcal{H})$ be the standard operator of type $(11,10$,

$9,7,6,5)$. Let $u=e_{14}+e_{34}+e_{43}, v=e_{25}+e_{35}+e_{54}$. Let

$$
\mathcal{M}_{0}=\vee\left\{e_{11}, e_{21}, e_{31}, e_{22}, e_{32}, e_{33}, e_{51}, u, T u, T^{2} u, T^{3} u, v, T v, T^{2} v, T^{3} v, T^{4} v\right\}
$$

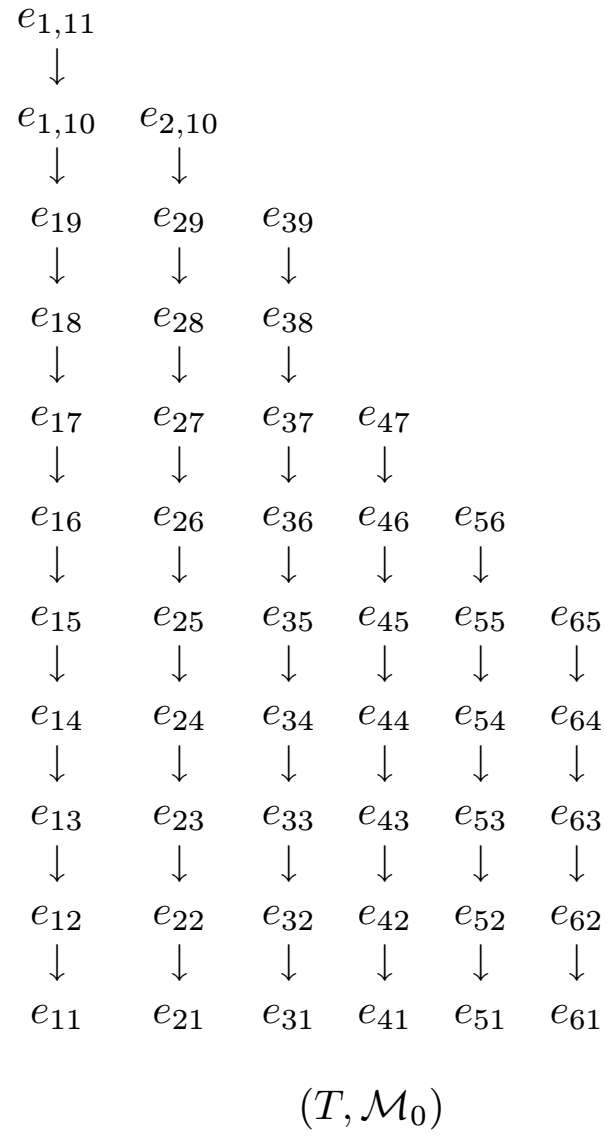

Figure 5.5 
Then

$$
\begin{aligned}
T^{10} \mathcal{H} & =\vee\left\{e_{11}\right\}, \\
T\left(\mathcal{M}_{0} \cap T^{8} \mathcal{H}\right) & =\vee\left\{e_{21}\right\}, \\
T^{2}\left(\mathcal{M}_{0} \cap T^{6} \mathcal{H}\right) & =\vee\left\{e_{31}\right\}, \\
T^{3}\left(\mathcal{M}_{0} \cap T^{4} \mathcal{H}\right) & =\vee\left\{e_{11}+e_{31}\right\}, \\
T^{4}\left(\mathcal{M}_{0} \cap T^{2} \mathcal{H}\right) & =\vee\left\{e_{21}+e_{31}\right\} .
\end{aligned}
$$

The previous lemma for $a=e_{11}, b=e_{21}$ and $c=e_{31}$ gives that the lattice $\mathcal{L}_{T, \mathcal{M}_{0}}$ is infinite.

For $k=0,1, \ldots$, set $w_{k}=e_{16}-k e_{26}+e_{6,5}$. Denote $\mathcal{M}_{k}=\mathcal{M}_{0} \vee\left\{T^{j} w_{k}\right.$ : $j \geq 0\}$. Then (5.1) remains true with $\mathcal{M}_{0}$ replaced by $M_{k}$ and $T^{5} \mathcal{M}_{k}=\vee\left\{e_{11}-k e_{21}\right\}$. Suppose $k \neq l$ and $\left(T, \mathcal{M}_{k}\right) \stackrel{\operatorname{dim}}{\sim}\left(T, \mathcal{M}_{l}\right)$. Let $\phi: \mathcal{L}_{T, \mathcal{M}_{k}} \rightarrow \mathcal{L}_{T, \mathcal{M}_{l}}$ be a $(\mathrm{T}, \mathrm{T})$-lattice isomomorphism satisfying $\phi\left(\mathcal{M}_{k}\right)=\phi\left(\mathcal{M}_{l}\right)$. Since (5.1) is true for $\mathcal{M}_{0}$ replaced by either $\mathcal{M}_{k}$ or $\mathcal{M}_{l}$, Lemma 5.11 and (5.1) imply $\phi\left(\vee\left\{e_{11}-j e_{21}\right\}\right)=\vee\left\{e_{11}-j e_{21}\right\}$ for every $j$. Thus for $j \neq k, \phi(\{0\})=\phi\left(\vee\left\{e_{11}-k e_{21}\right\} \cap \vee\left\{e_{11}-j e_{21}\right\}\right)=\phi\left(T^{5} M_{k} \cap \vee\left\{e_{11}-\right.\right.$ $\left.\left.j e_{21}\right\}\right)=T^{5} M_{j} \cap\left(\vee\left\{e_{11}-j e_{21}\right\}\right)=\vee\left\{e_{11}-j e_{21}\right\}$, which is a contradiction with the assumption that $\phi$ preserves dimensions.

We conclude with a conjecture.

Conjecture 5.13. The number of dim-equivalence classes is countable.

Acknowledgements. The first named author is supported by grant DMS9303702 from the National Science Foundation. The second named author wishes to express his gratitude to the School of Mathematics of Georgia Institute of Technology for its kind hospitality while this paper was written. He was partially supported by grant 201/96/0411 of GA ČR.

\section{REFERENCES}

[1] O. Azenhas and E. Marques de Sa, Matrix realizations of Littlewood-Richardson sequences, Lin. Mult. Alg. 27 (1990), 229-242.

[2] H. Bercovici, The quasisimilarity orbits of invariant subspaces, J. Funct. Anal. 95 (1991), 344-363. 
[3] H. Bercovici and A. Tannenbaum, The invariant subspaces of a uniform Jordan operator, J. Math. Anal. Appl. 156 (1991), 220-230.

[4] I. Gohberg and M. A. Kaashoek, Unsolved problems in matrix and operator theory, II. Partial multiplicities of a product, Integral Equations Operator Theory 2 (1979), 116-120.

[5] I. Gohberg, P. Lancaster, and L. Rodman Invariant Subspaces of Matrices with applications, Wiley-Interscience, New York 1986.

[6] J. Green, Symmetric functions and p-modules, Lecture notes, Manchester, 1961.

[7] T. Klein, The multiplication of Schur-functions and extensions of p-modules, J. London Math. Soc. 43 (1968), 280-284.

[8] I. G. Macdonald, Symmetric functions and Hall polynomials, Oxford University Press, New York, 1995.

[9] P. McMullen and G. C. Shephard, Convex polytopes and the upper bound conjecture, London Math. Soc. Lecture Note Series 3, Cambridge University Press, 1971.

[10] L. Rodman and M. Schaps, On the partial multiplicities of a product of two matrix polynomials, Integral Equations Operator Theory 2 (1979), 564-598

[11] G. Ph. A. Thijsse, Rules for the partial multiplicities of the product of holomorphic matrix functions, Integral Equations Operator Theory 3 (1980), 515-528.

[12] G. Ph. A. Thijsse, The local invariant factors of a product of holomorphic matrix functions: the order 4 case, Integral Equations Operator Theory 16 (1993), $277-302$.

School of Mathematics

Georgia Institute of Technology

Atlanta, Georgia 30332

U.S.A.

e-mail: li@math.gatech.edu
Institute of Mathematics

Academy of Sciences of the Czech Republic

11567 Prague 1, Žitná 25

Czech Republic

e-mail: vmuller@mbox.cesnet.cz 